\title{
Expression of Heterosis, Gene Action and Relationship among Morpho-physiological and Yield Characters in Sunflower under Different Levels of Water Supply Awaad , H. A.; A. H. Salem; M. M. A. Ali and K.Y. Kamal \\ Crop Sci. Dept., Fac. Agric., Zagazig Univ., Egypt \\ Corresponding author: A.H. Salem Email : zasalem@yahoo.com
}

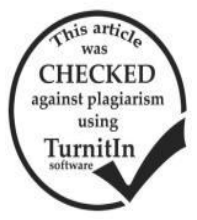

\section{ABSTRACT}

In order to monitor heterosis, inheritance and interrelationship among morpho-physiological and yield characters in sunflower, half diallel crosses among seven genetically divergent inbred lines were evaluated under adequate water supply, moderate and severe stress. Drought sensitivity index indicated that the parental sunflower genotypes L38 and L990 and the $\mathrm{F}_{1}$ crosses L38 x L990 and L350 x L460 were more tolerant to water stress, whereas L11 and L235 and the $\mathrm{F}_{1}$ cross L11 x L990 classified as sensitive one. Cross combination L38 x L350 scored desired and significant heterosis for leaf chlorophyll content at moderate stress; transpiration rate at severe stress; achene yield/plant and achene oil content at adequate water supply and moderate stress. Additive gene action had a great role in controlling transpiration rate, plant height and achene oil content, while dominance was important in controlling achene yield/plant under the three levels of water regimes. The environmental variance had significant effect on gene expression of physiological and yield characters in most cases. Narrow sense heritability was high $(>50 \%)$ for transpiration rate and low $(<30 \%)$ for achene yield/plant under the three levels of water regimes. Significant positive correlations were registered between achene yield/plant and each of leaf water content, transpiration rate, plant height, head diameter and 100-achene weight across three environments. The maximum direct effect on achene yield/plant was accounted for transpiration rate and plant height with values of $12.941 \%$ and $12.219 \%$, respectively. The highest indirect effects on achene yield/plant variation were observed for transpiration rate via plant height followed by transpiration rate via 100-achene weight; leaf water content via 100-achene weight with values of $8.442 \%, 5.530 \%$ and $4.579 \%$, respectively across three environments.

Keywords: Sunflower, heterosis, gene action, correlation, path analysis, water stress

\section{INTRODUCTION}

Egypt's production of edible vegetable oils has been suffering several problems due to the lower domestic production of oil crops that resulted in failing to meet the needs of domestic consumption (Hassan and Sahfique, 2010). Sunflower (Helianthus annuus L.) has become an important oil crop in the world. The total area reached about 24.8 million hectares worldwide with average productivity 1.66 ton/ha. gave total production 41.3 million metric tons. Meanwhile, in Egypt, the total area was about 10000 hectares with average productivity 2.5 ton/ha. gave total production 25000 tons (FAOSTAT, 2014).

Sunflower breeders have therefore devoted effort to develop superior genotypes for seed yield and adaptation to the different stress factors. Drought was a key factor responsible for yield losses of up to $20 \%$ (Reddy et al., 2004). High yield is the ultimate objective of any breeding program. However, high yield and drought tolerance are two different mechanisms that are often found to oppose each other. For achieving this purpose, information on the performance of parents, their behavior in hybrid combinations is prerequisite. Therefore, estimate of performance, heterosis, genetic components is essential for identification the promising hybrids tolerant to drought in breeding programs.

The importance of additive and non-additive gene action for seed yield and other related characters have been mentioned by many investigators of them, Goksoy and Turan (2004) indicated that non-additive gene action was accounted the most part of the genetic variation for seed yield and plant height. Neither additive nor non-additive variances were found to be significant for head diameter and 1000- seed weight. However, Ortis et al. (2005) indicated the predominant role of additive component for plant height, 1000-seed weight and seed oil content. 1000seed weight and oil yield were under control of both additive and dominant effects, plant height and oil content were controlled by additive effects, however over dominant effects were detected for seed yield (Ghaffari et al., 2011). Also, Burli et al. (2001) and Ravi et al. (2004) discussed the importance of non-additive gene effects in the expression of seed yield and all contributing characters. Furthermore, Bakheit et al. (2010) indicated that the dominance gene action (non-additive gene affects) was more important in controlling plant height, head diameter, 100 -achen weight, achene yield/plant and achene oil \%.

Utilization of heterosis has allowed sunflower to become one of the major oil seed in many countries of the world. One of approximately 16.5 million hectares of sunflower grown in the major producing countries, 11.5 million hectares are planted to hybrids (Miller, 1998). The introduction of hybrid cultivars produced an increase in yield potential around $25 \%$ through exploitation of heterosis (Fernández-Martínez et al. 2009). Encheva et al., (2015) recorded positive and significant heterosis in the direction of both relative to parental average and relative to better parent for plant height, diameter of head and seed yield per plant.

Yield is a complex character and is a function of several traits and their interaction with environment. It is important to measure the mutual relationship between various plant attributes and determine the characters, on which selection procedure can be based for direct and indirect genetic improvement of crop yield. The estimates of genotypic, phenotypic and environmental correlations among the characters are useful in planning the selection strategies. Since, the relations between leaf chlorophyll content, relative water content, transpiration in particular during water stress, are well described in sunflower (Hirasawa et al., 1995; Guidi and Soldatini, 1997; Pankovic et al., 1999 and Pourmohammad, 2016). However, only a few papers reported the genetic determinism of these traits of them Hervé et al. (2001)

Path coefficient analysis is helpful in partitioning the correlation coefficients into its direct and indirect 
effects, and many researchers calculated path coefficient analysis of them, Rauf et al. (2012), Ardiarini et al. (2013) and Iqbal et al. (2013). Also, Darvishzadeh et al. (2011) found that genotypic correlations manifest that seed yield per plant was positively and significantly associated with head diameter, plant height and achene traits at well-watered condition and with head diameter and chlorophyll content at water-stressed state. Head diameter and number of achene at both conditions and chlorophyll content at water-stressed condition have positive direct effect on seed yield/plant.

The present study was conducted to estimate heterosis, genetic components, correlations and path coefficient analyses for leaf water content, leaf chlorophyll value, transpiration rate, plant height, head diameter, 100achene weight, achene yield/plant and achene oil content $(\%)$ in 7 inbred lines and their $F_{1}$ crosses.

\section{MATERIALS AND METHODS}

\section{Description of the studied sunflower genotypes}

Field experiments were carried out during the two successive seasons 2009 and 2010 at El-Khattara Agriculture Research Stations, Faculty of Agriculture, Zagazig University, Egypt. The experimental materials comprised 7 sunflower inbred lines (L38 and L11 from Egypt and L350, L460, L990, L770 and L235 from Bulgaria). The seeds of all inbred lines were obtained from Oil Research Department, Field Crops Research Institute, Agricultural Research Center (ARC), Ministry of Agriculture and Land Reclamation, Egypt.

\section{Mating system and experimental layout}

In 2009 season, the seven inbred lines were crossed in $7 \times 7$ half diallel to obtain sufficient seed for evaluation in the next season. Each inbred line was sown in 10 rows; each row was 6 meters length, plant to plant and row to row distances were 30 and $50 \mathrm{~cm}$, respectively. In 2010 season, the resultant 21 hybrids were evaluated along with their respective parents under three levels of water regimes (adequate water supply with $7140 \mathrm{~m}^{3}$, moderate $4760 \mathrm{~m}^{2}$ and severe stress 2380 $\mathrm{m}^{3} / \mathrm{ha}$.). Quantities of water irrigation were adjusted using a water counter for all irrigation treatments under drip irrigation system. A split plot design with four replicates was used, where the main plots assigned for water regimes and the subplots for sunflower genotypes. The subplot area was $15 \mathrm{~m}^{2}$ and comprised two rows for each parent and $F_{1}$ hybrid. The row length was $5 \mathrm{~m}$, with spacing $50 \mathrm{~cm}$ between rows. Three seeds of sunflower genotypes were sown in hills $30 \mathrm{~cm}$ apart on $1^{\text {st }}$ June in both seasons. After 21 days from sowing, thinning to one plant/hill was done. All the other cultural practices for growing sunflower were applied as recommended. The soil of the experimental site is sandy in texture and had an average $\mathrm{pH}$ of 8.1 and organic matter content of $0.26 \%$. The average available $\mathrm{N}, \mathrm{P}, \mathrm{K}$ contents were 15.1, 3.2 and 90.5 ppm, respectively.

\section{Collected data}

Plant height $(\mathrm{cm})$, leaf chlorophyll content, leaf water content $(\%)$, transpiration rate, head diameter $(\mathrm{cm})$, 100- achene weigh $(\mathrm{g})$, achene yield/plant $(\mathrm{g})$ and achene oil content (\%) were estimated for each sunflower genotype of each replicate under the levels of water regime. At flowering stage, five randomly selected plants were taken from each entry of each replication to estimate leaf chlorophyll content, transpiration rate and leaf water content. Leaf chlorophyll content (SPAD values) was assessed using chlorophyll meter (SPAD - 502, Minolta), measurements were taken from three points of each leaf (upper, middle and lower part). The average of these three readings was considered as SPAD reading of the leaf. Leaf transpiration rate $\left(\mathrm{mg} \mathrm{H}_{2} \mathrm{O} / \mathrm{cm}^{2} / \mathrm{h}\right)$ was estimated according to the adopted rapid weighing systems (Migahid and Amer, 1950 and Gosev, 1960). Leaf water content (\%) was determined according to Turner (1981). At harvest, five guarded plants were taken from each entry of each replication for estimating plant height, head diameter and achene yield/ plant. Drought sensitivity index (DSI) was calculated according to Fischer and Maurer (1978). DSI = $[1-(\mathrm{Ys} / \mathrm{Yp}] / \mathrm{SI}$, while SI (stress intensity) $=1-(\overline{\mathrm{Y}} \mathrm{s} /$ $\bar{Y} p$ ). Where, Ys and Yp are the achene yield of a genotype under stress and adequate water supply conditions, respectively, also $\bar{Y} s$ and $\bar{Y} p$ are general mean yield in stress and non-stress conditions, respectively. A sample of 100 - filled seeds (at $8 \%$ moisture content) was drawn at random from the bulked seeds of 5 plants with an electronic balance. Achene oil content \% was determined according to AOAC (1984) using Soxhlet apparatus and diethyl ether as a solvent.

\section{Statistical analysis}

The obtained data were analyzed according to Steel and Torrie (1980). Mid-parent ( $\overline{\mathbf{M}} . \overline{\mathbf{P}}$.) heterosis value was evaluated by using t-test according to Wynne et al. (1970). Genetic components and derived parameters were estimated using diallel biometrical approach outlined by Hayman (1954a and b). Genotypic, phenotypic and environmental correlations and path coefficient analyses were computed according to Miller et al. (1958). The path coefficient analysis was estimated as outlined by Dewey and Lu (1959). A PC Microsoft Excel and SAS 9.1® Computer program for Windows were used for statistical analysis.

\section{RESULTS AND DISCUSSION}

\section{Analysis of variance}

Analysis of variance of sunflower genotypes (G) i.e., parents and their $F_{1}$ crosses and between the three levels of water regime $(\mathrm{W})$ and $(\mathrm{GX} \mathrm{W})$ interaction were computed for leaf water content, leaf chlorophyll content (SPAD), transpiration rate $\left(\mathrm{mg} \mathrm{H}_{2} \mathrm{O} / \mathrm{cm}^{2} / \mathrm{h}\right)$, plant height $(\mathrm{cm})$, head diameter $(\mathrm{cm}), 100$-achene weight $(\mathrm{g})$, achene yield/plant $(\mathrm{g})$ and achene oil content $(\%)$. The results showed highly significant differences between parental sunflower genotypes and their $F_{1}$ crosses under the three levels of water supply and $(\mathrm{GX}$ W) interaction for the studied characters. This confirms the presence of considerable amount of genetic variability among the genetic materials valid for further biometrical assessments. It is interest to note that, the sunflower genotypes differed in their behavior from adequate water supply, moderate to severe water stress. Significant differences were detected between sunflower parents and their $F_{1}$ crosses under different environments for morpho-physiological 
characters by Herve et al. (2001) and Ibrahim et al. (2003) and for yield contributing characters by Rauf and Sadaqat (2007) and Iqbal et al. (2013).

\section{Mean performance}

\section{Physiological characters}

The results in Table (1) indicated that highly significant differences were observed between parents and their $F_{1}$ crosses under the three levels of water regimes for various physiological characters. The parental sunflower genotypes $\mathrm{P}_{3}$ (L11) and $\mathrm{P}_{4}$ (L460) exhibited the highest values for leaf water content, as well as their $\mathrm{F}_{1}$ crosses $\mathrm{P}_{3} \times \mathrm{P}_{4}$ and $\mathrm{P}_{4} \times \mathrm{P}_{7}$ under adequate water supply; $\mathrm{P}_{2}$ (L350) and $\mathrm{P}_{4}$ (L460) as well as $\mathrm{F}_{1}$ crosses $\mathrm{P}_{1} \times \mathrm{P}_{2}, \mathrm{P}_{1} \times \mathrm{P}_{7}, \mathrm{P}_{2} \times \mathrm{P}_{3}, \mathrm{P}_{2} \times \mathrm{P}_{4}, \mathrm{P}_{2} \times \mathrm{P}_{5}$, $\mathrm{P}_{3} \times \mathrm{P}_{4}, \mathrm{P}_{3} \times \mathrm{P}_{5}$ and $\mathrm{P}_{4} \times \mathrm{P}_{7}$ under moderate as well as $\mathrm{P}_{1}$ and $\mathrm{P}_{2}$ and their $\mathrm{F}_{1}$ crosses $\mathrm{P}_{1} \times \mathrm{P}_{2}, \mathrm{P}_{1} \times \mathrm{P}_{4}, \mathrm{P}_{2} \times \mathrm{P}_{4}, \mathrm{P}_{2} \times$ $\mathrm{P}_{6}$ and $\mathrm{P}_{4} \times \mathrm{P}_{7}$ under severe stress. The general mean of leaf water content tended to decrease from 91.00, 82.46 and $68.56 \%$ for adequate water supply, moderate and severe stress, respectively.
Results of leaf chlorophyll content indicated that the highest values were registered by $\mathrm{P}_{1}$ (L38) and $\mathrm{P}_{4}$ (L460) as well as $\mathrm{F}_{1}$ crosses $\mathrm{P}_{1} \times \mathrm{P}_{2}, \mathrm{P}_{1} \times \mathrm{P}_{4}, \mathrm{P}_{2} \times \mathrm{P}_{4}, \mathrm{P}_{3}$ x $\mathrm{P}_{4}$ and $\mathrm{P}_{4} \times \mathrm{P}_{6}$ under adequate water supply; $\mathrm{P}_{1}$ (L38), $\mathrm{P}_{4}$ (L460) as well as $\mathrm{F}_{1}$ crosses $\mathrm{P}_{1} \times \mathrm{P}_{2}, \mathrm{P}_{1} \times \mathrm{P}_{3}, \mathrm{P}_{1} \times \mathrm{P}_{4}$, $\mathrm{P}_{2} \times \mathrm{P}_{3}, \mathrm{P}_{3} \times \mathrm{P}_{4}$ and $\mathrm{P}_{3} \times \mathrm{P}_{7}$ under moderate as well as $\mathrm{P}_{1}$ (L38), $\mathrm{P}_{4}$ (L460) and $\mathrm{P}_{7}$ (L235) and $\mathrm{F}_{1}$ crosses $\mathrm{P}_{1} \times \mathrm{P}_{4}$, $\mathrm{P}_{2} \times \mathrm{P}_{3}, \mathrm{P}_{2} \times \mathrm{P}_{4}, \mathrm{P}_{3} \times \mathrm{P}_{4}, \mathrm{P}_{4} \times \mathrm{P}_{6}$ and $\mathrm{P}_{4} \times \mathrm{P}_{7}$ under severe stress. The general mean of leaf chlorophyll valued 37.61, 38.01 and 36.94 under adequate water supply, moderate and severe stress, respectively.

Lower transpiration rates were registered by $\mathrm{P}_{1}$ (L38) and $\mathrm{P}_{4}$ (L460) as well as the $\mathrm{F}_{1}$ crosses $\mathrm{P}_{1} \times \mathrm{P}_{3}, \mathrm{P}_{1}$ x $\mathrm{P}_{4}, \mathrm{P}_{1} \times \mathrm{P}_{7}, \mathrm{P}_{2} \times \mathrm{P}_{4}, \mathrm{P}_{3} \times \mathrm{P}_{4}, \mathrm{P}_{3} \times \mathrm{P}_{7}$ and $\mathrm{P}_{4} \times \mathrm{P}_{7}$ under adequate water supply; $\mathrm{P}_{1}$ and $\mathrm{P}_{3}$ as well as the $\mathrm{F}_{1}$ crosses $\mathrm{P}_{1} \times \mathrm{P}_{2}, \mathrm{P}_{1} \times \mathrm{P}_{3}, \mathrm{P}_{1} \times \mathrm{P}_{7}, \mathrm{P}_{2} \times \mathrm{P}_{7}, \mathrm{P}_{3} \times \mathrm{P}_{7}$ and $\mathrm{P}_{4} \times \mathrm{P}_{7}$ under moderate, as well as $\mathrm{P}_{1}$ and $\mathrm{P}_{7}$ and the $\mathrm{F}_{1}$ crosses $\mathrm{P}_{1} \times \mathrm{P}_{6}, \mathrm{P}_{1} \times \mathrm{P}_{7}, \mathrm{P}_{2} \times \mathrm{P}_{7}, \mathrm{P}_{3} \times \mathrm{P}_{6}, \mathrm{P}_{3} \times \mathrm{P}_{7}, \mathrm{P}_{5} \times \mathrm{P}_{7}$ and $\mathrm{P}_{6} \times$ $\mathrm{P}_{7}$ under severe stress.

Table 1. Mean performance for physiological characters of sunflower genotypes of half-diallel analysis under three environments.

\begin{tabular}{|c|c|c|c|c|c|c|c|c|c|}
\hline \multirow{2}{*}{$\begin{array}{l}\text { Character } \\
\text { Water supply } \\
\text { Genotype }\end{array}$} & \multicolumn{3}{|c|}{ Leaf water content (\%) } & \multicolumn{2}{|c|}{ Leaf chlorophyll content } & \multirow{2}{*}{$\begin{array}{c}\text { (SPAD) } \\
\text { Severe } \\
\text { stress }\end{array}$} & \multicolumn{3}{|c|}{ Transpiration rate $\left(\mathrm{mg} \mathrm{H}_{2} \mathrm{O} / \mathrm{cm}^{2} / \mathrm{h}\right)$} \\
\hline & $\begin{array}{c}\text { Adequate } \\
\text { water supply }\end{array}$ & $\begin{array}{c}\text { Moderate } \\
\text { stress }\end{array}$ & $\begin{array}{l}\text { Severe } \\
\text { stress }\end{array}$ & $\begin{array}{c}\text { Adequate } \\
\text { water supply }\end{array}$ & $\begin{array}{c}\text { Moderate } \\
\text { stress }\end{array}$ & & $\begin{array}{c}\text { Adequate } \\
\text { water supply }\end{array}$ & $\begin{array}{c}\text { Moderate } \\
\text { stress }\end{array}$ & $\begin{array}{l}\text { Severe } \\
\text { stress }\end{array}$ \\
\hline P1 (L38) & 87.73 & 78.05 & 70.02 & 41.18 & 39.03 & 36.60 & 0.68 & 0.32 & 0.30 \\
\hline P2 (L350) & 91.05 & 89.35 & 70.53 & 38.38 & 36.85 & 35.43 & 0.94 & 0.72 & 0.45 \\
\hline P3 (L11) & 91.40 & 77.15 & 66.58 & 38.20 & 36.55 & 35.88 & 0.86 & 0.65 & 0.35 \\
\hline P4 (L460) & 90.25 & 82.10 & 68.13 & 40.38 & 40.85 & 40.53 & 0.78 & 0.70 & 0.58 \\
\hline P5 (L990) & 89.10 & 80.23 & 64.88 & 36.98 & 34.58 & 35.45 & 1.12 & 0.80 & 0.40 \\
\hline P6 (L770) & 90.18 & 79.25 & 64.98 & 36.98 & 37.00 & 35.70 & 1.08 & 0.87 & 0.32 \\
\hline P7 (L235) & 91.20 & 80.63 & 65.85 & 38.15 & 35.73 & 36.93 & 0.88 & 0.57 & 0.25 \\
\hline $\mathrm{P} 1 \times \mathrm{P} 2$ & 94.60 & 85.40 & 72.60 & 39.45 & 39.25 & 37.50 & 0.90 & 0.65 & 0.40 \\
\hline P1 x P3 & 90.03 & 80.43 & 70.25 & 37.40 & 39.68 & 36.83 & 0.80 & 0.62 & 0.36 \\
\hline $\mathrm{P} 1 \times \mathrm{P} 4$ & 91.13 & 82.23 & 73.35 & 38.45 & 39.05 & 39.18 & 0.82 & 0.68 & 0.51 \\
\hline P1 x P5 & 89.98 & 79.43 & 69.60 & 37.95 & 38.15 & 35.50 & 0.98 & 0.75 & 0.38 \\
\hline P1 x P6 & 92.33 & 79.45 & 72.28 & 37.00 & 38.50 & 36.05 & 0.96 & 0.80 & 0.35 \\
\hline P1 x P7 & 87.83 & 80.53 & 71.25 & 36.48 & 39.03 & 35.03 & 0.85 & 0.52 & 0.30 \\
\hline $\mathrm{P} 2 \times \mathrm{P} 3$ & 92.00 & 84.65 & 69.80 & 36.80 & 40.80 & 38.80 & 0.92 & 0.70 & 0.42 \\
\hline $\mathrm{P} 2 \times \mathrm{P} 4$ & 92.30 & 86.53 & 69.15 & 38.33 & 38.38 & 39.35 & 0.88 & 0.71 & 0.54 \\
\hline P2 x P5 & 90.25 & 87.58 & 69.80 & 36.00 & 37.00 & 37.03 & 1.02 & 0.78 & 0.43 \\
\hline $\mathrm{P} 2 \times \mathrm{P} 6$ & 92.53 & 85.35 & 70.50 & 36.98 & 38.05 & 36.10 & 1.05 & 0.85 & 0.42 \\
\hline P2 x P7 & 90.18 & 83.68 & 69.48 & 35.88 & 39.03 & 35.30 & 0.93 & 0.60 & 0.35 \\
\hline $\mathrm{P} 3 \times \mathrm{P} 4$ & 92.73 & 82.30 & 69.28 & 39.65 & 40.00 & 41.63 & 0.84 & 0.70 & 0.50 \\
\hline P3 x P5 & 89.60 & 87.28 & 66.80 & 36.18 & 38.08 & 36.05 & 0.99 & 0.76 & 0.38 \\
\hline $\mathrm{P} 3 \times \mathrm{P} 6$ & 90.80 & 85.58 & 65.98 & 36.95 & 36.18 & 36.73 & 0.96 & 0.84 & 0.34 \\
\hline P3 x P7 & 89.40 & 80.53 & 66.58 & 36.00 & 40.95 & 36.00 & 0.89 & 0.65 & 0.32 \\
\hline P4 x P5 & 92.68 & 85.35 & 67.05 & 37.03 & 37.15 & 37.00 & 0.95 & 0.76 & 0.49 \\
\hline P4 x P6 & 93.53 & 81.30 & 69.95 & 41.48 & 37.48 & 38.98 & 0.90 & 0.82 & 0.45 \\
\hline P4 x P7 & 93.08 & 82.30 & 70.83 & 36.18 & 36.15 & 38.20 & 0.85 & 0.62 & 0.40 \\
\hline P5 x P6 & 89.20 & 80.48 & 61.53 & 34.98 & 37.13 & 36.20 & 1.00 & 0.83 & 0.35 \\
\hline P5 x P7 & 91.70 & 81.63 & 65.38 & 36.83 & 37.15 & 35.10 & 0.99 & 0.72 & 0.32 \\
\hline P6 x P7 & 91.38 & 80.30 & 67.33 & 36.80 & 36.50 & 35.40 & 1.02 & 0.77 & 0.30 \\
\hline Mean & 91.00 & 82.46 & 68.56 & 37.61 & 38.01 & 36.94 & 0.92 & 0.71 & 0.39 \\
\hline L.S.D $0.05(\mathrm{G})$ & 3.42 & 6.28 & 3.71 & 2.10 & 3.50 & 3.41 & 0.08 & 0.07 & 0.06 \\
\hline L.S.D $0.05(\mathrm{~W})$ & & 0.87 & & & 0.57 & & & 0.01 & \\
\hline L.S.D $0.05(\mathrm{Gx} \mathrm{W})$ & & 4.60 & & & 3.04 & & & 0.07 & \\
\hline
\end{tabular}

The general mean of transpiration rate appeared to be decreased with increasing water stress and valued $0.92,0.71$ and $0.39 \mathrm{mg} \mathrm{H} \mathrm{H}_{2} \mathrm{O} / \mathrm{cm}^{2} / \mathrm{h}$ under adequate water supply, moderate and severe stress, respectively. The interaction between sunflower genotypes and water supply treatments was significant, hereby the studied materials are effected by water stress applications

Some sunflower genotypes showed relative stability from environment to another i.e. $P_{2}(\mathrm{~L} 350)$ and the $F_{1}$ crosses $\mathrm{P}_{2} \times \mathrm{P}_{5}$ and $\mathrm{P}_{3} \times \mathrm{P}_{5}$ for leaf water content from 
7140 to $4760 \mathrm{~m}^{3}$ water supply; $\mathrm{P}_{2}, \mathrm{P}_{3}, \mathrm{P}_{4}$, and $\mathrm{P}_{7}$, as well as the $\mathrm{F}_{1}$ crosses $\mathrm{P}_{2} \times \mathrm{P}_{3}, \mathrm{P}_{2} \times \mathrm{P}_{4}, \mathrm{P}_{2} \times \mathrm{P}_{5}, \mathrm{P}_{3} \times \mathrm{P}_{6}, \mathrm{P}_{4} \times \mathrm{P}_{5}, \mathrm{P}_{4} \times$ $\mathrm{P}_{6}$ and $\mathrm{P}_{4} \times \mathrm{P}_{4}$ for leaf chlorophyll content as well as $\mathrm{P}_{1}$ and their $F_{1}$ cross $P_{1} \times P_{4}$ for transpiration rate from 4760 to $2380 \mathrm{~m}^{3}$. Therefore, these genotypes are more tolerant to water stress. The grand mean of both characters tended to decrease from adequate water supply, moderate to severe stress. This result could be due to drought acclimation affected the partitioning of water between the apoplastic and symplastic fractions (Maury et al. 2006), they also found differential responses of three sunflower genotypes to water stress for leaf water parameters i.e., predawn leaf water potential, photosynthetic rate and stomatal conductance.

Yield and its attributes

The results in Tables (2 and 3) indicated that, $P_{1}$ (L38) and $\mathrm{P}_{3}$ (L11) were the shortest ones among the studied parents, as well as their $\mathrm{F}_{1}$ crosses $\mathrm{P}_{1} \times \mathrm{P}_{3}$ and $\mathrm{P}_{1} \times \mathrm{P}_{4}$. On the other hand, $\mathrm{P}_{5}$ (L990) and $\mathrm{P}_{6}$ (L770) were the tallest ones, as well as their $\mathrm{F}_{1}$ crosses $\mathrm{P}_{2} \times \mathrm{P}_{5}$,
$\mathrm{P}_{2} \times \mathrm{P}_{6}, \mathrm{P}_{4} \times \mathrm{P}_{5}$ and $\mathrm{P}_{5} \times \mathrm{P}_{6}$. This trend was hold true under the three levels of water supply. Plant height tended to decrease from 137.87 to 123.92 and $103.34 \mathrm{~cm}$ under $7140 \mathrm{~m}^{3}, 4760 \mathrm{~m}^{3}$ and $2380 \mathrm{~m}^{3}$, respectively, as a result of water stress effect on elongation of cells.

Parental sunflower genotypes $\mathrm{P}_{4}$ (L460), $\mathrm{P}_{5}$ (L990) and $\mathrm{P}_{6}$ (L770) gave broader heads which transmitted to their $\mathrm{F}_{1}$ progenies $\mathrm{P}_{2} \times \mathrm{P}_{4}, \mathrm{P}_{2} \times \mathrm{P}_{5}, \mathrm{P}_{2} \times$ $\mathrm{P}_{6}, \mathrm{P}_{3} \times \mathrm{P}_{6}, \mathrm{P}_{5} \times \mathrm{P}_{6}$ and $\mathrm{P}_{6} \times \mathrm{P}_{7}$ under the three environments. Otherwise, $\mathrm{P}_{1}$ and $\mathrm{P}_{2}$ as well as their $\mathrm{F}_{1}$ crosses $\mathrm{P}_{1} \times \mathrm{P}_{2}, \mathrm{P}_{1} \times \mathrm{P}_{3}, \mathrm{P}_{1} \times \mathrm{P}_{6}$ and $\mathrm{P}_{2} \times \mathrm{P}_{7}$ were narrow in head diameter among the studied sunflower genotypes. The value of head diameter tended to decrease from 19.8 to 16.36 and $14.21 \mathrm{~cm}$, for adequate water supply, moderate to severe stress, respectively.

Sunflower inbreds $\mathrm{P}_{5}$ (L990) and $\mathrm{P}_{6}$ (L770) gave the heaviest 100-achene weight, as well as the $F_{1}$ crosses $P_{2} x$ $\mathrm{P}_{6}, \mathrm{P}_{3} \times \mathrm{P}_{5}, \mathrm{P}_{4} \times \mathrm{P}_{5}, \mathrm{P}_{4} \times \mathrm{P}_{6}, \mathrm{P}_{5} \times \mathrm{P}_{6}$ and $\mathrm{P}_{6} \times \mathrm{P}_{7}$ rather than the studied genotypes under the three environments.

Table 2. Mean performance for morphological characters of sunflower genotypes of half-diallel analysis under three environments

\begin{tabular}{|c|c|c|c|c|c|c|c|c|c|}
\hline \multirow{2}{*}{$\begin{array}{l}\text { Character } \\
\text { Water supply } \\
\text { Genotype }\end{array}$} & \multicolumn{3}{|c|}{ Plant height (cm) } & \multicolumn{3}{|c|}{ Head diameter $(\mathrm{cm})$} & \multicolumn{3}{|c|}{ 100-achene weight (g) } \\
\hline & $\begin{array}{c}\text { Adequate } \\
\text { water supply }\end{array}$ & $\begin{array}{c}\text { Moderate } \\
\text { stress }\end{array}$ & $\begin{array}{c}\text { Severe } \\
\text { stress }\end{array}$ & $\begin{array}{c}\text { Adequate } \\
\text { water supply }\end{array}$ & $\begin{array}{c}\text { Moderate } \\
\text { stress }\end{array}$ & $\begin{array}{c}\text { Severe } \\
\text { stress }\end{array}$ & $\begin{array}{c}\text { Adequate } \\
\text { water supply }\end{array}$ & $\begin{array}{c}\text { Moderate } \\
\text { stress }\end{array}$ & $\begin{array}{c}\text { Severe } \\
\text { stress }\end{array}$ \\
\hline P1 (L38) & 79.95 & 68.38 & 60.50 & 15.80 & 13.28 & 12.38 & 9.35 & 7.48 & 5.38 \\
\hline P2 (L350) & 130.88 & 119.28 & 98.53 & 16.68 & 13.50 & 12.70 & 10.40 & 9.23 & 6.00 \\
\hline P3 (L11) & 114.85 & 100.63 & 91.98 & 18.69 & 16.78 & 12.98 & 8.53 & 7.65 & 6.20 \\
\hline P4 (L460) & 120.23 & 117.75 & 96.98 & 19.28 & 15.30 & 14.03 & 8.25 & 7.28 & 7.00 \\
\hline P5 (L990) & 150.55 & 129.48 & 112.20 & 19.03 & 17.00 & 15.13 & 8.85 & 8.03 & 8.23 \\
\hline P6 (L770) & 148.63 & 138.45 & 104.73 & 18.88 & 16.70 & 15.08 & 8.95 & 7.80 & 7.30 \\
\hline P7 (L235) & 141.00 & 128.20 & 105.08 & 18.25 & 15.33 & 13.95 & 8.15 & 7.63 & 7.08 \\
\hline $\mathrm{P} 1 \times \mathrm{P} 2$ & 134.83 & 126.93 & 91.10 & 17.80 & 15.93 & 12.58 & 11.08 & 8.35 & 6.38 \\
\hline $\mathrm{P} 1 \times \mathrm{P} 3$ & 115.30 & 106.25 & 100.40 & 19.13 & 16.03 & 12.55 & 8.88 & 8.43 & 6.85 \\
\hline $\mathrm{P} 1 \times \mathrm{P} 4$ & 115.18 & 102.88 & 92.20 & 18.73 & 17.10 & 14.65 & 8.70 & 8.33 & 7.85 \\
\hline $\mathrm{P} 1 \times \mathrm{P} 5$ & 142.85 & 130.38 & 100.55 & 18.30 & 15.03 & 13.23 & 9.98 & 8.90 & 7.48 \\
\hline $\mathrm{P} 1 \times \mathrm{P} 6$ & 142.08 & 117.23 & 83.55 & 17.45 & 14.60 & 12.90 & 9.78 & 9.33 & 7.78 \\
\hline $\mathrm{P} 1 \times \mathrm{P} 7$ & 142.75 & 133.00 & 106.63 & 19.33 & 15.28 & 13.98 & 9.45 & 8.10 & 7.60 \\
\hline $\mathrm{P} 2 \times \mathrm{P} 3$ & 134.23 & 127.18 & 96.95 & 18.75 & 16.33 & 15.28 & 10.03 & 9.13 & 6.70 \\
\hline $\mathrm{P} 2 \times \mathrm{P} 4$ & 126.55 & 121.00 & 101.43 & 21.30 & 17.10 & 14.85 & 10.70 & 8.85 & 7.28 \\
\hline $\mathrm{P} 2 \times \mathrm{P} 5$ & 152.00 & 134.75 & 111.58 & 20.68 & 16.55 & 14.95 & 10.48 & 10.00 & 6.88 \\
\hline $\mathrm{P} 2 \times \mathrm{P} 6$ & 155.13 & 137.43 & 117.31 & 22.33 & 17.45 & 14.75 & 11.33 & 9.10 & 6.75 \\
\hline $\mathrm{P} 2 \times \mathrm{P} 7$ & 150.10 & 125.34 & 112.25 & 17.83 & 14.70 & 13.40 & 8.70 & 8.98 & 7.30 \\
\hline P3 x P4 & 125.68 & 114.28 & 98.38 & 20.83 & 16.23 & 14.38 & 9.78 & 8.25 & 6.68 \\
\hline P3 x P5 & 140.83 & 134.28 & 115.45 & 21.43 & 17.53 & 14.58 & 10.43 & 9.13 & 6.73 \\
\hline P3 x P6 & 136.60 & 131.13 & 111.90 & 22.50 & 16.90 & 13.33 & 8.75 & 7.25 & 6.50 \\
\hline P3 x P7 & 147.70 & 125.50 & 113.90 & 19.58 & 16.10 & 14.15 & 8.83 & 8.25 & 6.68 \\
\hline $\mathrm{P} 4 \times \mathrm{P} 5$ & 153.45 & 128.65 & 110.55 & 21.18 & 18.00 & 15.65 & 9.08 & 8.28 & 7.15 \\
\hline $\mathrm{P} 4 \times \mathrm{P} 6$ & 149.30 & 136.23 & 113.60 & 21.75 & 16.73 & 15.98 & 9.05 & 7.95 & 6.90 \\
\hline $\mathrm{P} 4 \times \mathrm{P} 7$ & 155.95 & 136.28 & 109.10 & 21.18 & 16.85 & 14.68 & 8.88 & 7.08 & 6.98 \\
\hline P5 x P6 & 156.60 & 133.78 & 112.00 & 23.43 & 19.13 & 15.50 & 9.60 & 8.88 & 7.38 \\
\hline P5 x P7 & 149.75 & 130.75 & 112.93 & 21.90 & 18.33 & 14.63 & 9.23 & 7.05 & 6.05 \\
\hline P6 x P7 & 147.45 & 134.43 & 111.68 & 22.38 & 18.30 & 15.83 & 9.60 & 7.80 & 7.25 \\
\hline Mean & 137.87 & 123.92 & 103.34 & 19.80 & 16.36 & 14.21 & 9.46 & 8.30 & 6.94 \\
\hline L.S.D $0.05(G)$ & 12.58 & 12.32 & 11.00 & 1.86 & 1.80 & 1.44 & 0.86 & 1.20 & 1.34 \\
\hline L.S.D $0.05(\mathrm{~W})$ & & 2.24 & & & 0.32 & & & 0.22 & \\
\hline L.S.D $0.05(\mathrm{G} \times \mathrm{W})$ & & 11.86 & & & 1.69 & & & 1.14 & \\
\hline
\end{tabular}

The best genotypes had high performance for achene yield/plant were $\mathrm{P}_{4}$ (L460) followed by $\mathrm{P}_{7}$ (L235) and $\mathrm{P}_{6}$ (L770) along with the $\mathrm{F}_{1}$ crosses $\mathrm{P}_{1} \times \mathrm{P}_{3}, \mathrm{P}_{1} \times \mathrm{P}_{6}, \mathrm{P}_{2}$ $\times \mathrm{P}_{4}, \mathrm{P}_{2} \times \mathrm{P}_{5}, \mathrm{P}_{4} \times \mathrm{P}_{5}$ and $\mathrm{P}_{4} \times \mathrm{P}_{6}$ under adequate water supply; $\mathrm{P}_{4}$ and $\mathrm{P}_{5}$ as well as the $\mathrm{F}_{1}$ crosses $\mathrm{P}_{1} \times \mathrm{P}_{2}, \mathrm{P}_{1} \times \mathrm{P}_{6}$ and $\mathrm{P}_{4} \times \mathrm{P}_{5}$ under moderate, as well as $\mathrm{P}_{4}$ and $\mathrm{P}_{5}$ and the $\mathrm{F}_{1}$ crosses $\mathrm{P}_{1} \times \mathrm{P}_{6}$ and $\mathrm{P}_{4} \times \mathrm{P}_{6}$ under severe stress (Table 3). These results coupled with heterotic effects, where these crosses displayed positive and significant heterosis. Achene yield/plant tended to decrease by decreasing water supply from 7140 to 4760 and $2380 \mathrm{~m}^{2} / \mathrm{ha}$. with values of $80.05,67.46$ and $54.56 \mathrm{~g}$, respectively.

Furthermore, the parental sunflower genotypes $\mathrm{P}_{1}$ (L38) followed by $P_{5}$ (L990) and $P_{7}$ (L235) gave the highest achene oil content and their $\mathrm{F}_{1}$ crosses $\mathrm{P}_{1} \times \mathrm{P}_{3}, \mathrm{P}_{1} \times \mathrm{P}_{5}, \mathrm{P}_{1} \mathrm{x}$ $\mathrm{P}_{7}, \mathrm{P}_{3} \times \mathrm{P}_{5}$ and $\mathrm{P}_{5} \times \mathrm{P}_{7}$. The value of achene oil content 
showed relative stable under the three levels of water supply in parental sunflower genotypes $\mathrm{P}_{2}$ (L350) and $\mathrm{P}_{7}$ (L235) and the $\mathrm{F}_{1}$ crosses $\mathrm{P}_{2} \times \mathrm{P}_{3}, \mathrm{P}_{2} \times \mathrm{P}_{4}, \mathrm{P}_{3} \times \mathrm{P}_{5}, \mathrm{P}_{3} \times \mathrm{P}_{7}, \mathrm{P}_{5} \times \mathrm{P}_{7}$ and $\mathrm{P}_{6} \times \mathrm{P}_{7}$. This result could be discussed on the basis that achene oil content was more heritable character and less influenced by the environmental conditions.

These results could be discussed with reference to the connection between transpiration efficiency and plant growth accompanied by changes in plant growth characteristics and the effect of drought stress on partitioning of current assimilates between reproductive and non-reproductive organs as indicated by Virgona et al. (1990). Similar observation was also confirmed by Darvishzadeh et al. (2011), Ardiarini et al. (2013) and Iqbal et al. (2013). Also, Ibrahim et al. (2003) detected significant variation between sunflower genotypes in chlorophyll content and yield attributes. They added that Fodac cultivar was superior in chlorophyll content, plant height, head diameter, 100-achene weight, seed yield/ plant and oil yield/plant compared with Erflor and Malbar.

\section{Drought sensitivity index (DSI)}

Drought sensitivity index (DSI) as regards of achene yield/plant was estimated for determining tolerance of sunflower genotypes to water stress (Table 3). Genotypes with DSI values less than 1.0 are more tolerant to water stress, while those with values above 1.0 are sensitive one. Therefore under severe stress, $\mathrm{P}_{1}$ (L38) and $\mathrm{P}_{5}$ (L990) and the $\mathrm{F}_{1}$ crosses $\mathrm{P}_{1} \times \mathrm{P}_{5}, \mathrm{P}_{1} \times \mathrm{P}_{6}, \mathrm{P}_{1} \times \mathrm{P}_{7}, \mathrm{P}_{2} \times \mathrm{P}_{7}, \mathrm{P}_{3} \times \mathrm{P}_{4}, \mathrm{P}_{3} \times$ $\mathrm{P}_{5}, \mathrm{P}_{5} \times \mathrm{P}_{7}$ and $\mathrm{P}_{6} \times \mathrm{P}_{7}$ exhibited DSI less than unity, hence these genotypes were considered as more tolerant to severe stress. Particularly, parental genotypes $\mathrm{P}_{1}$ (L38) and $\mathrm{P}_{5}$ (L990) and their $F_{1}$ cross $P_{1} \times P_{5}$ gave the smallest and desirable DSI with values $0.63,0.68$ and 0.79 , respectively. On the other side, sunflower cross $\mathrm{P}_{2} \times \mathrm{P}_{5}$ exhibited DSI value more than $1.0(1.32)$, it is classified as sensitive to water stress. Moreover, for moderate stress, $\mathrm{P}_{1}$ (L38), $\mathrm{P}_{2}$ (L350), $P_{4}$ (L460), $P_{5}$ (L990) and $P_{6}$ (L770) and the $F_{1}$ crosses $\mathrm{P}_{1} \times \mathrm{P}_{2}, \mathrm{P}_{1} \times \mathrm{P}_{3}, \mathrm{P}_{1} \times \mathrm{P}_{6}, \mathrm{P}_{1} \times \mathrm{P}_{7}, \mathrm{P}_{2} \times \mathrm{P}_{6}, \mathrm{P}_{3} \times \mathrm{P}_{4}, \mathrm{P}_{3} \times$ $\mathrm{P}_{5}$ and $\mathrm{P}_{5} \times \mathrm{P}_{6}$ had DSI less than unity, hence these genotypes were considered as more tolerant to moderate stress. Furthermore, the remaining genotypes attained DSI values near 1.0 and considered as moderate tolerant to water stress. These findings are in close agreement with those of Rauf and Sadqat (2007).

Table 3. Achene yield / plant, achene oil \% and drought susceptibility index (DSI) of sunflower genotypes under three environments

\begin{tabular}{|c|c|c|c|c|c|c|c|c|}
\hline \multirow{3}{*}{$\begin{array}{l}\text { Character } \\
\text { Water supply } \\
\text { Genotype }\end{array}$} & \multicolumn{5}{|c|}{ Achene yield /plant (g) } & \multicolumn{3}{|c|}{ Achene oil content $(\%)$} \\
\hline & \multirow{2}{*}{$\begin{array}{c}\text { Adequate } \\
\text { water supply }\end{array}$} & \multirow{2}{*}{$\begin{array}{l}\text { Moderate } \\
\text { stress }\end{array}$} & \multirow[b]{2}{*}{$\begin{array}{r}\text { Severe } \\
\text { stress }\end{array}$} & \multicolumn{2}{|c|}{ (DSI) } & \multirow[b]{2}{*}{$\begin{array}{l}\text { Adequate water } \\
\text { supply }\end{array}$} & \multirow{2}{*}{$\begin{array}{c}\text { Moderate } \\
\text { stress }\end{array}$} & \multirow{2}{*}{$\begin{array}{c}\text { Severe } \\
\text { stress }\end{array}$} \\
\hline & & & & $\begin{array}{c}\text { Moderate } \\
\text { stress }\end{array}$ & $\begin{array}{c}\text { Severe } \\
\text { Stress }\end{array}$ & & & \\
\hline P1 (L38) & 50.33 & 43.80 & 40.28 & 0.82 & 0.63 & 36.10 & 35.40 & 34.90 \\
\hline P2 (L350) & 76.78 & 67.23 & 48.73 & 0.79 & 1.15 & 23.40 & 23.10 & 23.00 \\
\hline P3 (L11) & 74.85 & 62.80 & 53.18 & 1.02 & 0.91 & 29.50 & 27.90 & 27.10 \\
\hline P4 (L460) & 85.73 & 74.68 & 55.10 & 0.82 & 1.12 & 25.40 & 24.80 & 24.50 \\
\hline P5 (L990) & 79.10 & 75.63 & 61.85 & 0.28 & 0.68 & 30.50 & 29.50 & 28.80 \\
\hline P6 (L770) & 80.20 & 68.05 & 53.28 & 0.96 & 1.05 & 27.40 & 26.20 & 25.60 \\
\hline P7 (L235) & 81.30 & 61.40 & 51.13 & 1.56 & 1.17 & 30.40 & 29.80 & 29.10 \\
\hline $\mathrm{P} 1 \times \mathrm{P} 2$ & 81.55 & 75.50 & 54.93 & 0.47 & 1.03 & 32.50 & 31.20 & 30.40 \\
\hline P1 x P3 & 84.15 & 73.40 & 52.93 & 0.81 & 1.17 & 35.20 & 33.50 & 32.60 \\
\hline $\mathrm{P} 1 \times \mathrm{P} 4$ & 79.83 & 67.53 & 54.53 & 0.98 & 1.00 & 32.10 & 30.10 & 28.53 \\
\hline P1 x P5 & 64.48 & 51.83 & 48.20 & 1.25 & 0.79 & 36.40 & 35.40 & 34.10 \\
\hline P1 x P6 & 84.50 & 76.45 & 60.43 & 0.61 & 0.89 & 30.50 & 29.20 & 27.20 \\
\hline P1 x P7 & 77.73 & 67.30 & 56.55 & 0.85 & 0.86 & 38.30 & 34.40 & 32.20 \\
\hline $\mathrm{P} 2 \times \mathrm{P} 3$ & 83.23 & 68.40 & 59.30 & 1.13 & 0.90 & 28.40 & 27.00 & 28.40 \\
\hline $\mathrm{P} 2 \times \mathrm{P} 4$ & 83.95 & 69.20 & 53.90 & 1.12 & 1.12 & 26.80 & 26.10 & 25.20 \\
\hline $\mathrm{P} 2 \times \mathrm{P} 5$ & 97.10 & 68.15 & 56.25 & 1.90 & 1.32 & 28.60 & 27.50 & 25.60 \\
\hline $\mathrm{P} 2 \times \mathrm{P} 6$ & 77.93 & 72.33 & 51.45 & 0.46 & 1.07 & 28.80 & 25.40 & 24.20 \\
\hline $\mathrm{P} 2 \times \mathrm{P} 7$ & 77.18 & 62.03 & 55.73 & 1.25 & 0.87 & 31.20 & 29.00 & 28.80 \\
\hline P3 x P4 & 78.78 & 69.58 & 58.40 & 0.74 & 0.81 & 29.90 & 27.40 & 26.10 \\
\hline P3 x P5 & 77.80 & 68.23 & 55.90 & 0.78 & 0.88 & 31.20 & 29.10 & 29.20 \\
\hline P3 x P6 & 74.80 & 63.50 & 52.15 & 0.96 & 0.95 & 29.40 & 27.80 & 28.30 \\
\hline P3 x P7 & 82.03 & 62.63 & 54.55 & 1.50 & 1.05 & 30.30 & 30.00 & 29.50 \\
\hline P4 x P5 & 87.60 & 75.70 & 55.95 & 0.86 & 1.13 & 30.30 & 29.90 & 28.00 \\
\hline P4 x P6 & 93.80 & 70.58 & 60.58 & 1.57 & 1.11 & 26.70 & 25.00 & 24.60 \\
\hline P4 x P7 & 81.85 & 67.45 & 53.38 & 1.12 & 1.09 & 30.40 & 29.10 & 27.30 \\
\hline P5 x P6 & 82.70 & 73.25 & 51.78 & 0.73 & 1.17 & 31.10 & 30.20 & 28.00 \\
\hline P5 x P7 & 80.70 & 67.23 & 57.88 & 1.06 & 0.89 & 30.60 & 29.10 & 30.10 \\
\hline P6 x P7 & 81.58 & 64.95 & 59.55 & 1.30 & 0.85 & 29.80 & 29.60 & 28.60 \\
\hline Mean & 80.05 & 67.46 & 54.56 & & & 30.40 & 29.03 & 28.21 \\
\hline L.S.D $0.05(G)$ & 13.94 & 10.94 & 10.81 & & & 0.91 & 1.17 & 1.39 \\
\hline L.S.D $0.05(\mathrm{~W})$ & & 2.24 & & & & & 0.22 & \\
\hline L.S.D ${ }_{0.05}(\mathrm{G} \times \mathrm{W})$ & & 11.86 & & & & & 1.16 & \\
\hline
\end{tabular}

\section{Heterosis
Physiological characters}

Results presented in Table (4) show heterosis as percentage of mid-parents for leaf water content, leaf chlorophyll content and transpiration rate. Cross combinations $\mathrm{P}_{1} \times \mathrm{P}_{2}, \mathrm{P}_{1} \times \mathrm{P}_{6}, \mathrm{P}_{4} \times \mathrm{P}_{5}$ and $\mathrm{P}_{4} \times \mathrm{P}_{6}$ gave significant positive heterotic effects for leaf water content at adequate water supply. Meanwhile, no significant 
positive heterosis was found among all crosses at both moderate and severe stress treatments. Also, significant positive heterosis was found for leaf chlorophyll content in the $\mathrm{F}_{1}$ crosses $\mathrm{P}_{1} \times \mathrm{P}_{2}$ and $\mathrm{P}_{4} \times \mathrm{P}_{6}$ at adequate water supply; $\mathrm{P}_{1} \times \mathrm{P}_{3}, \mathrm{P}_{2} \times \mathrm{P}_{3}, \mathrm{P}_{2} \times \mathrm{P}_{7}$ and $\mathrm{P}_{3} \times \mathrm{P}_{7}$ at moderate and $\mathrm{P}_{2} \times \mathrm{P}_{3}$ and $\mathrm{P}_{3} \times \mathrm{P}_{4}$ at severe drought stress.

On the other hand, desirable significantly negative heterosis has been registered for transpiration rate by only one cross $\left(\mathrm{P}_{5} \times \mathrm{P}_{6}\right)$ at adequate water supply; $\mathrm{P}_{1} \times \mathrm{P}_{7}, \mathrm{P}_{2} \mathrm{x}$ $\mathrm{P}_{3}, \mathrm{P}_{2} \times \mathrm{P}_{4}, \mathrm{P}_{2} \times \mathrm{P}_{5}, \mathrm{P}_{2} \times \mathrm{P}_{7}, \mathrm{P}_{3} \times \mathrm{P}_{4}, \mathrm{P}_{3} \times \mathrm{P}_{5}, \mathrm{P}_{3} \times \mathrm{P}_{7}, \mathrm{P}_{5} \times$ $\mathrm{P}_{6}$ and $\mathrm{P}_{5} \times \mathrm{P}_{7}$ at moderate and all crosses at severe stress. These results were desired for the breeder where the genotypes, which maintain high levels of leaf water content with high ability to reduce transpiration rate might be considered as tolerant to water stress. Hervé et al. (2001) found that the mean values of recombinant inbred lines were intermediate between the two parents for leaf chlorophyll concentration, net photosynthesis, internal $\mathrm{CO}_{2}$ concentration and transpiration. Some recombinant inbred lines had more extreme values than the parents showing a transgressive segregation.

Yield and its attributes
Results presented in Tables (5 and 6) clear showed negative and highly significant heterosis of mid-parents for plant height in the cross $\left(\mathrm{P}_{5} \times \mathrm{P}_{6}\right)$ only at moderate water stress, as well as $\mathrm{P}_{1} \times \mathrm{P}_{6}, \mathrm{P}_{2} \times \mathrm{P}_{3}, \mathrm{P}_{2} \times \mathrm{P}_{4}, \mathrm{P}_{2} \times \mathrm{P}_{5}$, $\mathrm{P}_{5} \times \mathrm{P}_{6}, \mathrm{P}_{5} \times \mathrm{P}_{7}$ and $\mathrm{P}_{6} \times \mathrm{P}_{7}$ at severe stress. This result might be due to accumulation of decreasing alleles controlling short plants. Otherwise, positive and highly significant heterosis were recorded for plant height in 14 out of 21 cross ranging from $7.43 \%$ for $\left(\mathrm{P}_{2} \times \mathrm{P}_{5}\right)$ to $22.61 \%$ for $\left(\mathrm{P}_{1} \times \mathrm{P}_{7}\right)$ at adequate water supply; 7 out of 21 cross varied from $8.85 \%$ for $\left(\mathrm{P}_{4} \times \mathrm{P}_{7}\right)$ to $21.75 \%$ for $\left(\mathrm{P}_{1}\right.$ $\left.\mathrm{x} \mathrm{P}_{7}\right)$ at moderate and 2 out of 21 cross valued $13.24 \%$ for $\left(\mathrm{P}_{1} \times \mathrm{P}_{7}\right)$ and $14.38 \%$ for $\left(\mathrm{P}_{1} \times \mathrm{P}_{3}\right)$ at severe stress.

Significant or highly significant positive midparent heterosis have been recorded for head diameter by the cross combinations $\left(\mathrm{P}_{1} \times \mathrm{P}_{3}, \mathrm{P}_{1} \times \mathrm{P}_{7}, \mathrm{P}_{2} \times \mathrm{P}_{4}, \mathrm{P}_{2} \times\right.$ $\mathrm{P}_{5}, \mathrm{P}_{2} \times \mathrm{P}_{6}, \mathrm{P}_{3} \times \mathrm{P}_{4}, \mathrm{P}_{3} \times \mathrm{P}_{5}, \mathrm{P}_{3} \times \mathrm{P}_{6}, \mathrm{P}_{4} \times \mathrm{P}_{6}, \mathrm{P}_{4} \times \mathrm{P}_{7}, \mathrm{P}_{5} \times$ $\mathrm{P}_{6}$ and $\left.\mathrm{P}_{6} \times \mathrm{P}_{7}\right)$ at adequate water supply; $\left(\mathrm{P}_{1} \times \mathrm{P}_{4}\right)$ at moderate water stress. Also, 100-achene weight exhibited significant positive heterosis for eight crosses $\left(\mathrm{P}_{1} \times \mathrm{P}_{2}, \mathrm{P}_{1} \times \mathrm{P}_{5}, \mathrm{P}_{2} \times \mathrm{P}_{4}, \mathrm{P}_{2} \times \mathrm{P}_{5}, \mathrm{P}_{2} \times \mathrm{P}_{6}, \mathrm{P}_{3} \times \mathrm{P}_{4}, \mathrm{P}_{3} \times\right.$ $\mathrm{P}_{5}$ and $\mathrm{P}_{6} \times \mathrm{P}_{7}$ ) at adequate water supply only.

Table 4. Heterosis over mid-parent (M.P.) for physiological characters of sunflower crosses of half-diallel analysis in three environments.

\begin{tabular}{|c|c|c|c|c|c|c|c|c|c|}
\hline \multirow{2}{*}{$\begin{array}{l}\text { Character } \\
\text { Water supply } \\
\text { Genotype }\end{array}$} & \multicolumn{3}{|c|}{ Leaf water content $(\%)$} & \multicolumn{2}{|c|}{ Leaf chlorophyll content } & \multirow{2}{*}{$\begin{array}{c}\text { (SPAD) } \\
\text { Severe } \\
\text { stress }\end{array}$} & \multicolumn{3}{|c|}{ Transpiration rate $\left(\mathrm{mg} \mathrm{H}_{2} \mathrm{O} / \mathrm{cm}^{2} / \mathrm{h}\right)$} \\
\hline & $\begin{array}{c}\text { Adequate } \\
\text { water supply }\end{array}$ & $\begin{array}{c}\text { Moderate } \\
\text { stress }\end{array}$ & $\begin{array}{c}\text { Severe } \\
\text { stress }\end{array}$ & $\begin{array}{c}\text { Adequate } \\
\text { water supply }\end{array}$ & $\begin{array}{c}\text { Moderate } \\
\text { stress }\end{array}$ & & $\begin{array}{c}\text { Adequate } \\
\text { water supply }\end{array}$ & $\begin{array}{c}\text { Moderate } \\
\text { stress }\end{array}$ & $\begin{array}{l}\text { Severe } \\
\text { stress }\end{array}$ \\
\hline $\mathrm{P} 1 \times \mathrm{P} 2$ & 5.51 & -3.67 & $-8.99^{* * \pi}$ & $4.97^{*}$ & 6.43 & 3.97 & 10.00 & -7.69 & $-41.25^{3 / 6}$ \\
\hline P1 x P3 & 0.51 & -2.50 & $-9.82^{* *}$ & 0.00 & $7.81^{*}$ & 1.60 & 3.75 & -7.26 & $-45.07^{* * *}$ \\
\hline $\mathrm{P} 1 \times \mathrm{P} 4$ & 2.35 & -3.27 & $-6.24^{* * *}$ & -0.10 & 0.83 & 1.56 & $10.98^{* *}$ & -1.47 & $-23.53^{* *}$ \\
\hline P1 x P5 & 1.74 & -5.73 & $-9.63^{* *}$ & 3.06 & 6.72 & -1.48 & $8.16^{*}$ & 1.33 & $-42.11^{* *}$ \\
\hline $\mathrm{P} 1 \times \mathrm{P} 6$ & $3.66^{*}$ & -5.08 & $-5.64^{*}$ & 0.57 & 4.42 & -0.28 & $8.33^{*}$ & 3.13 & $-44.93^{* *}$ \\
\hline P1 x P7 & -1.86 & -4.53 & $-7.77^{* *}$ & -2.47 & 7.34 & -4.96 & $8.24^{*}$ & $-20.19^{* *}$ & $-55.00^{* *}$ \\
\hline P2 x P3 & 0.84 & 0.65 & $-12.91^{* * *}$ & -0.03 & $11.80^{* * *}$ & $8.12^{*}$ & 2.17 & $-13.57^{* *}$ & $-53.57^{* *}$ \\
\hline $\mathrm{P} 2 \times \mathrm{P} 4$ & 1.79 & -0.06 & $-15.09^{* * *}$ & 1.11 & 0.62 & 3.49 & 2.27 & $-15.49^{* *}$ & $-40.74^{* * *}$ \\
\hline P2 x P5 & 0.19 & 2.21 & $-11.69^{* *}$ & -0.56 & 5.41 & 4.29 & -0.98 & $-11.54^{* *}$ & $-55.81^{* *}$ \\
\hline $\mathrm{P} 2 \times \mathrm{P} 6$ & 2.07 & 0.23 & $-10.66^{* * *}$ & 2.10 & 4.83 & 1.49 & 3.81 & -6.47 & $-50.00^{* * *}$ \\
\hline $\mathrm{P} 2 \times \mathrm{P} 7$ & -1.05 & -2.58 & $-12.92^{* * *}$ & -2.54 & $8.84^{*}$ & -2.48 & 2.15 & $-25.83^{* *}$ & $-70.00^{* * *}$ \\
\hline $\mathrm{P} 3 \times \mathrm{P} 4$ & 2.05 & -5.41 & $-15.14^{* * *}$ & 3.85 & 4.09 & $8.23^{*}$ & 2.38 & $-11.43^{* *}$ & $-44.00^{* * *}$ \\
\hline P3 x P5 & -0.73 & 1.68 & $-16.97^{* *}$ & -0.69 & 7.49 & 1.07 & 0.00 & $-9.21^{*}$ & $-65.79^{* * *}$ \\
\hline P3 x P6 & 0.01 & 0.29 & $-18.51^{* * *}$ & 1.42 & -0.73 & 2.55 & -1.04 & -2.67 & $-73.53^{* *}$ \\
\hline P3 x P7 & -2.13 & $-6.81^{*}$ & $-18.10^{* *}$ & -2.81 & $12.58^{* *}$ & -1.11 & 2.25 & $-10.00^{* * *}$ & $-73.44^{* *}$ \\
\hline $\mathrm{P} 4 \times \mathrm{P} 5$ & $3.24^{*}$ & 0.13 & $-15.68^{* * *}$ & -4.66 & -1.08 & -2.67 & 0.00 & -3.95 & $-21.65^{* *}$ \\
\hline $\mathrm{P} 4 \times \mathrm{P} 6$ & $3.54^{*}$ & -4.24 & $-10.95^{* *}$ & $6.57^{*}$ & -3.44 & 2.21 & -3.33 & -0.61 & $-22.22^{* *}$ \\
\hline $\mathrm{P} 4 \times \mathrm{P} 7$ & 2.52 & -3.81 & $-10.20^{* *}$ & $-8.74^{*}$ & -5.46 & -1.37 & 2.35 & -8.87 & $-28.75^{* * *}$ \\
\hline P5 x P6 & -0.49 & -4.60 & $-25.21^{* * *}$ & -3.54 & 2.42 & 1.73 & $-10.00^{* *}$ & $-19.88^{* * *}$ & $-105.71^{\text {*** }}$ \\
\hline P5 x P7 & 1.69 & -3.97 & $-18.51^{* * *}$ & 0.07 & 4.21 & -3.10 & -1.01 & $-17.36^{* * *}$ & $-114.06^{* * *}$ \\
\hline P6 x P7 & 0.75 & -6.35 & $-15.87^{* *}$ & -0.34 & 2.16 & -2.58 & 3.92 & -7.14 & $-121.67^{* * *}$ \\
\hline
\end{tabular}

*,** Significant at $\mathbf{P}=0.05$ and $\mathbf{P}=0.01$, respectively

Achene yield/plant revealed significantly positive heterosis in five crosses at adequate water supply, i.e., $\mathrm{P}_{1} \mathrm{x}$ $\mathrm{P}_{2}, \mathrm{P}_{1} \times \mathrm{P}_{3}, \mathrm{P}_{1} \times \mathrm{P}_{6}, \mathrm{P}_{1} \times \mathrm{P}_{7}$ and $\mathrm{P}_{2} \times \mathrm{P}_{5}$ ranging from $-2.03 \%$ for $\left(\mathrm{P}_{4} \times \mathrm{P}_{7}\right)$ to $25.62 \%$ for $\left(\mathrm{P}_{1} \times \mathrm{P}_{3}\right)$, as well as four crosses at moderate, i.e., $\mathrm{P}_{1} \times \mathrm{P}_{2}, \mathrm{P}_{1} \times \mathrm{P}_{3}, \mathrm{P}_{1} \times \mathrm{P}_{6}$ and $\mathrm{P}_{1} \times \mathrm{P}_{7}$ varied from $-12.52 \%$ for $\left(\mathrm{P}_{3} \times \mathrm{P}_{6}\right)$ to $22.94 \%$ for $\left(\mathrm{P}_{1} \times \mathrm{P}_{3}\right)$, but no significant heterosis was recorded for seed yield/plant at severe stress (Table 6), reinforcing the effect of water deficit on gene expression of yield character. For achene oil content, significant heterosis was detected in 17 out of 21 cross ranging from $-4.10 \%$ for $\left(\mathrm{P}_{1} \times \mathrm{P}_{6}\right)$ to $13.78 \%$ for $\left(\mathrm{P}_{2}\right.$ $\left.\mathrm{x} \mathrm{P}_{7}\right)$ at adequate water supply; 12 out of 21 cross ranging from $-6.68 \%$ for $\left(\mathrm{P}_{1} \times \mathrm{P}_{6}\right)$ to $8.28 \%$ for $\left(\mathrm{P}_{2} \times \mathrm{P}_{7}\right)$ at moderate and four out of 21 cross varied from $-13.42 \%$ for $\left(\mathrm{P}_{1} \times \mathrm{P}_{6}\right)$ to $11.09 \%$ for $\left(\mathrm{P}_{2} \times \mathrm{P}_{3}\right)$ at severe water stress.
Hence, on the basis of the obtained results, it could be concluded that the cross combination $\left(\mathrm{P}_{1} \times \mathrm{P}_{2}\right)$ showed desired heterosis for leaf water content, leaf chlorophyll content and 100-achene weight at adequate water supply, transpiration rate at severe stress; achene yield/plant and achene oil content at both adequate water supply and moderate stress. Also the cross combination $\left(\mathrm{P}_{1} \mathrm{x} \quad \mathrm{P}_{3}\right)$ showed significant positive heterosis for leaf chlorophyll content at moderate, head diameter at adequate water supply, achene yield/plant and achene oil content at both adequate water supply and moderate water stress conditions.

It is interest to note that the sunflower cross $\left(\mathrm{P}_{1} \times \mathrm{P}_{6}\right)$ produced the maximum value of heterosis for achene yield/plant under adequate water supply (22.77\%) and moderate $(22.58 \%)$ level with DSI value less than 1.0 and 
had significantly negative heterosis for plant height $(-10.52 \%)$ and transpiration rate $(-44.93 \%)$ at severe stress, therefore it could be considered the promising one and classified as tolerant to water stress. The present study suggested that the abovementioned crosses would be promising sources for commercial exploration of heterosis.

Table 5 . Heterosis over mid-parent (M.P.) for morphological characters of sunflower crosses of half-diallel analysis under three environments.

\begin{tabular}{|c|c|c|c|c|c|c|c|c|c|}
\hline \multirow{2}{*}{$\begin{array}{l}\text { Character } \\
\text { Water supply } \\
\text { Genotype }\end{array}$} & \multicolumn{3}{|c|}{ Plant height $(\mathrm{cm})$} & \multicolumn{3}{|c|}{ Head diameter $(\mathrm{cm})$} & \multicolumn{3}{|c|}{ 100-achene weight (g) } \\
\hline & $\begin{array}{c}\text { Adequate } \\
\text { water supply }\end{array}$ & $\begin{array}{c}\text { Moderate } \\
\text { stress }\end{array}$ & $\begin{array}{c}\text { Severe } \\
\text { stress }\end{array}$ & $\begin{array}{c}\text { Adequate } \\
\text { water supply }\end{array}$ & $\begin{array}{c}\text { Moderate } \\
\text { stress }\end{array}$ & $\begin{array}{c}\text { Severe } \\
\text { stress }\end{array}$ & $\begin{array}{c}\text { Adequate } \\
\text { water supply }\end{array}$ & $\begin{array}{c}\text { Moderate } \\
\text { stress }\end{array}$ & $\begin{array}{c}\text { Severe } \\
\text { stress }\end{array}$ \\
\hline $\mathrm{P} 1 \times \mathrm{P} 2$ & $21.82^{* * \pi}$ & $21.52^{3 * *}$ & 2.04 & 8.78 & 8.01 & $-13.32^{3 *}$ & $10.84^{3 * * *}$ & -11.23 & $-20.39^{*}$ \\
\hline P1 x P3 & $15.52^{* * *}$ & $15.02^{* * *}$ & $14.38^{* *}$ & $9.84^{*}$ & -1.64 & $-14.64^{* * *}$ & -0.70 & -0.89 & -13.50 \\
\hline $\mathrm{P} 1 \times \mathrm{P} 4$ & $13.10^{* * *}$ & 3.91 & 4.05 & 6.34 & $9.06^{*}$ & -1.79 & -1.15 & 0.15 & -4.14 \\
\hline P1 x P5 & $19.32^{* * *}$ & $19.68^{* * *}$ & 4.45 & 4.85 & $-9.15^{*}$ & $-16.92^{* *}$ & $8.77^{*}$ & 2.39 & $-17.56^{*}$ \\
\hline $\mathrm{P} 1 \times \mathrm{P} 6$ & $19.56^{* * *}$ & 6.85 & $-10.52^{* *}$ & 0.64 & $-11.30^{*}$ & $-19.67^{* * *}$ & 6.39 & 8.04 & -7.07 \\
\hline P1 x P7 & $22.61^{* * *}$ & $21.75^{* *}$ & $13.24^{* * *}$ & $11.90^{* * *}$ & -1.88 & -6.44 & 7.41 & -4.78 & -8.06 \\
\hline $\mathrm{P} 2 \times \mathrm{P} 3$ & $8.47^{*}$ & $8.98^{*}$ & $-14.93^{* *}$ & 5.70 & -2.45 & 2.95 & 5.61 & 1.10 & $-23.88^{* * *}$ \\
\hline $\mathrm{P} 2 \times \mathrm{P} 4$ & 0.79 & -2.74 & $-12.32^{* *}$ & $15.61^{* *}$ & 6.51 & -3.37 & $12.85^{* *}$ & 0.14 & $-19.59^{*}$ \\
\hline $\mathrm{P} 2 \times \mathrm{P} 5$ & $7.43^{*}$ & 3.40 & $-8.93^{*}$ & $13.66^{* * *}$ & -1.74 & -6.35 & $8.11^{*}$ & 7.88 & $-35.45^{* *}$ \\
\hline $\mathrm{P} 2 \times \mathrm{P} 6$ & $9.91^{\text {*** }}$ & 2.01 & -0.42 & $20.38^{* * *}$ & 4.37 & -7.63 & $14.57^{* *}$ & 0.00 & $-31.11^{* * *}$ \\
\hline $\mathrm{P} 2 \times \mathrm{P} 7$ & $9.44^{* *}$ & -3.35 & -5.10 & 2.03 & -8.84 & $-14.27^{* *}$ & -6.61 & -0.42 & $-19.69^{* * *}$ \\
\hline $\mathrm{P} 3 \times \mathrm{P} 4$ & 6.48 & -1.77 & -7.66 & $8.85^{*}$ & -4.74 & $-13.78^{* * *}$ & $14.19^{* *}$ & 4.24 & -16.29 \\
\hline P3 x P5 & 5.77 & $9.02^{*}$ & 1.67 & $11.99^{* *}$ & -1.82 & $-15.99^{* * *}$ & $16.67^{* *}$ & 9.32 & $-24.54^{\text {** }}$ \\
\hline P3 x P6 & 3.56 & 3.41 & 1.89 & $16.53^{* * *}$ & -4.70 & $-26.69^{* *}$ & 0.14 & -12.59 & $-21.73^{* *}$ \\
\hline P3 x P7 & $13.39^{* * *}$ & 3.17 & 3.46 & 5.65 & -5.63 & $-15.33^{* *}$ & 5.52 & 2.12 & -16.85 \\
\hline P4 x P5 & $11.77^{* * *}$ & 2.95 & -5.12 & $9.56^{*}$ & -0.76 & $-9.90^{*}$ & 5.79 & 1.66 & -15.21 \\
\hline $\mathrm{P} 4 \times \mathrm{P} 6$ & $9.96^{* * *}$ & 5.06 & 0.99 & $12.30^{* * *}$ & -7.55 & -7.51 & 4.97 & -0.94 & -12.68 \\
\hline P4 x P7 & $16.25^{* * *}$ & $8.85^{*}$ & -3.25 & $11.39^{* * *}$ & -2.67 & $-13.20^{\text {*** }}$ & 7.61 & -12.19 & -9.86 \\
\hline P5 x P6 & 4.48 & $-8.02^{*}$ & $-13.96^{* *}$ & $19.10^{* *}$ & 6.60 & $-10.00^{* *}$ & 7.29 & 6.20 & -9.49 \\
\hline P5 x P7 & 2.65 & -6.60 & $-13.18^{* *}$ & $14.90^{* * *}$ & 6.28 & $-12.74^{* *}$ & 7.86 & $-16.84^{*}$ & $-31.61^{* *}$ \\
\hline P6 x P7 & 1.79 & -2.97 & $-13.59^{* *}$ & $17.04^{* * *}$ & 6.56 & -3.71 & $10.94^{* *}$ & -6.25 & -10.52 \\
\hline
\end{tabular}

*** Significant at $\mathbf{P}=0.05$ and $\mathbf{P}=0.01$, respectively

Table 6. Heterosis over mid-parent (M.P.) for achene yield/head and achene oil content (\%) of sunflower crosses of half-diallel analysis under three environments.

\begin{tabular}{|c|c|c|c|c|c|c|}
\hline \multirow{2}{*}{$\begin{array}{l}\text { Character } \\
\text { Water supply } \\
\text { Genotype }\end{array}$} & \multicolumn{3}{|c|}{ Achene yield /plant (g) } & \multicolumn{3}{|c|}{ Achene oil content (\%) } \\
\hline & $\begin{array}{c}\text { Adequate water } \\
\text { supply }\end{array}$ & $\begin{array}{c}\text { Moderate } \\
\text { stress }\end{array}$ & $\begin{array}{r}\text { Severe } \\
\text { stress }\end{array}$ & $\begin{array}{c}\text { Adequate water } \\
\text { supply }\end{array}$ & $\begin{array}{c}\text { Moderate } \\
\text { stress }\end{array}$ & $\begin{array}{r}\text { Severe } \\
\text { stress }\end{array}$ \\
\hline $\mathrm{P} 1 \times \mathrm{P} 2$ & $22.07^{* * 3}$ & $22.15^{* * *}$ & 9.83 & $8.46^{* * *}$ & $5.13^{* *}$ & 2.80 \\
\hline P1 x P3 & $25.62^{* * *}$ & $22.94^{* * *}$ & 2.22 & $6.82^{* *}$ & $4.48^{* *}$ & 3.07 \\
\hline $\mathrm{P} 1 \times \mathrm{P} 4$ & 14.78 & 7.44 & 3.32 & $4.21^{\text {** }}$ & -1.16 & $-6.19^{* *}$ \\
\hline P1 x P5 & -0.37 & $-21.51^{* *}$ & -16.36 & $8.52^{* *}$ & $7.34^{\text {** }}$ & $4.84^{*}$ \\
\hline P1 x P6 & $22.77^{* *}$ & $22.58^{* *}$ & 14.27 & $-4.10^{* *}$ & $-6.68^{* *}$ & $-13.42^{* *}$ \\
\hline P1 x P7 & $15.33^{*}$ & $16.99^{*}$ & 10.30 & $13.19^{* * *}$ & $4.22^{* *}$ & -1.24 \\
\hline $\mathrm{P} 2 \times \mathrm{P} 3$ & 8.91 & -2.03 & -9.57 & $6.87^{* *}$ & $5.00^{* *}$ & $11.09^{* *}$ \\
\hline $\mathrm{P} 2 \times \mathrm{P} 4$ & 3.22 & -9.43 & $-22.33^{* *}$ & $8.96^{* * *}$ & $7.66^{* *}$ & $4.96^{*}$ \\
\hline $\mathrm{P} 2 \times \mathrm{P} 5$ & $19.73^{* * *}$ & -11.81 & $-23.22^{* *}$ & $5.77^{* *}$ & $3.82^{*}$ & -1.95 \\
\hline $\mathrm{P} 2 \times \mathrm{P} 6$ & -0.72 & -0.12 & $-26.38^{* *}$ & $11.81^{* * *}$ & 2.36 & -1.24 \\
\hline $\mathrm{P} 2 \times \mathrm{P} 7$ & -2.41 & -11.39 & -14.76 & $13.78^{* * *}$ & $8.28^{* * *}$ & $8.85^{* *}$ \\
\hline $\mathrm{P} 3 \times \mathrm{P} 4$ & -1.92 & -7.46 & -11.26 & $8.19^{* *}$ & 0.91 & -3.45 \\
\hline P3 x P5 & 1.06 & -10.27 & $-22.27^{* *}$ & $3.85^{* *}$ & -1.37 & 0.17 \\
\hline P3 x P6 & -3.64 & -12.52 & $-22.84^{* *}$ & $3.23^{*}$ & -0.18 & 2.65 \\
\hline P3 x P7 & 4.82 & -8.78 & -15.47 & 1.16 & 1.17 & 0.68 \\
\hline P4 x P5 & 5.92 & -6.57 & $-31.88^{* * *}$ & $7.76^{* *}$ & $8.19^{* *}$ & 3.21 \\
\hline P4 x P6 & 11.55 & -8.94 & -14.73 & 1.12 & -3.20 & -3.66 \\
\hline $\mathrm{P} 4 \times \mathrm{P} 7$ & -2.03 & -9.06 & $-28.20^{* *}$ & $8.22^{* * *}$ & $5.15^{\text {** }}$ & 0.18 \\
\hline P5 x P6 & 3.69 & -0.44 & $-27.84^{* * *}$ & $6.91^{\text {** }}$ & $6.13^{* *}$ & -0.18 \\
\hline P5 x P7 & 0.62 & -4.50 & -12.51 & 0.49 & $-3.61^{*}$ & 1.00 \\
\hline P6 x P7 & 1.01 & -9.01 & -10.26 & $3.02^{*}$ & $3.38^{*}$ & 1.22 \\
\hline
\end{tabular}

*,** Significant at $\mathbf{P}=0.05$ and $\mathbf{P}=0.01$, respectively

The magnitude of heterosis differed from adequate water supply, moderate to severe stress and tended to decrease with increasing water stress. Some crosses like $\mathrm{P}_{2}$ x $\mathrm{P}_{5}$ for achene yield/plant, plant height, 100-achene weight and achene oil content gave positive and highly significant heterosis under adequate water supply, but showed negative and insignificant heterosis at moderate level, as well as, negative and highly significant heterosis at severe water stress. Positive and negative heterosis over mid-parents were detected herein, may be due to the effect of environments on gene expression. Different magnitudes of heterosis for seed yield and various sunflower characters has also been registered by Gill et al. (1998), and Habib et al. (2007) and Bakhiet et al. (2010).

Genetic components and heritability

Physiological characters

Data presented in Table (7) indicated that the genetic components of variance and their derived parameters were fluctuated from adequate water supply, moderate to severe drought stress, since the dominance genetic variance was 
significant and accounted the main type controlling the inheritance of leaf water content under adequate water supply, resulting in an average degree of dominance $\left(\mathrm{H}_{1} / \mathrm{D}\right)^{0.5}$ was more than unity. Hereby, pedigree method might be exploited and superior genotypes could be identified from its phenotypic expression and selected in $\mathrm{F}_{2}$ On the otherwise, the additive genetic component was the prevailed type controlling leaf water content under moderate; leaf chlorophyll content under moderate and severe stress and transpiration rate under severe stress condition, resulting in an average degree of dominance $\left(\mathrm{H}_{1} / \mathrm{D}\right)^{0.5}$ was less than unity. In this connection, Hervé et al. (2001) revealed that additive gene action was more pronounced in controlling leaf chlorophyll concentration, net photosynthesis, internal $\mathrm{CO}_{2}$ concentration and transpiration rather than the environmental variance.

Furthermore, both additive (D) and dominance $\left(\mathrm{H}_{1}\right.$ and $\left.\mathrm{H}_{2}\right)$ genetic components were significant and involved in the inheritance of transpiration rate under moderate stress, with the predominant of additive gene action in controlling transpiration rate.

The covariance of additive and dominance gene effects in the parents ( $F$ value) was positive and significant for transpiration rate under adequate water supply and moderate stress, indicating that increasing alleles were more frequent than the decreasing ones in the parental populations. Whereas, in the remaining cases, the F values didn't reach the level of significance.

The overall dominance effects of heterozygous loci $\left(h^{2}\right)$ were positive and significant for leaf water content under the three levels of water regimes; transpiration rate under moderate and severe stress as well as leaf chlorophyll content under adequate water supply, hereby dominance was mainly attributable to heterozygous loci and seemed to be acting in positive direction. The environmental variance had significant effect on gene expression of physiological characters in most cases, explaining the changes in the genetic components and their derived parameters from condition to another.

The proportion of genes with positive and negative effects in the parents $\left(\mathrm{H}_{2} / 4 \mathrm{H}_{1}\right)$ was near to its maximum value $(0.25)$ for leaf water content under moderate stress and leaf chlorophyll content under adequate water supply. Whereas, the $\left(\mathrm{H}_{2} / 4 \mathrm{H}_{1}\right)$ was less than its maximum value $(0.25)$ for the remaining physiological characters under different levels of water regimes,

The proportion of dominance to recessive genes in the parents $(\mathrm{KD} / \mathrm{KR})$ was more than unity for leaf water content and leaf chlorophyll content under moderate stress; transpiration rate under normal and moderate water stress, showing an excess of dominant alleles in the parental populations. On the other wise, excess of recessive alleles have been detected for leaf water content and leaf chlorophyll content under both adequate water supply and severe stress as well as transpiration rate under severe stress only.

Narrow sense heritability $\left(T_{(n)}\right)$ which reflect the fixable type of gene action transmissible from the parents to the progeny was high $(>50 \%)$ for transpiration rate under the three levels of water regimes; leaf water content and leaf chlorophyll content under severe water stress. However, moderate narrow sense heritability have been registered for leaf water content under moderate water stress and leaf chlorophyll content under adequate water supply. Whereas, low narrow sense heritability was recorded for leaf water content under adequate water supply and leaf chlorophyll content under moderate water stress.

Table 7. Genetic components for physiological characters of sunflower genotypes of half-diallel analysis under three environments.

\begin{tabular}{|c|c|c|c|c|c|c|c|c|c|}
\hline \multirow{2}{*}{$\begin{array}{l}\text { Character } \\
\text { Water supply }\end{array}$} & \multicolumn{3}{|c|}{ Leaf water content (\%) } & \multicolumn{2}{|c|}{ Leaf chlorophyll content } & \multirow{2}{*}{$\begin{array}{c}\text { (SPAD) } \\
\text { Severe } \\
\text { stress } \\
\end{array}$} & \multicolumn{3}{|c|}{ Transpiration rate $\left(\mathrm{mg} \mathrm{H}_{2} \mathrm{O} / \mathrm{cm}^{2} / \mathrm{h}\right)$} \\
\hline & $\begin{array}{c}\text { Adequate } \\
\text { water supply }\end{array}$ & $\begin{array}{c}\text { Moderate } \\
\text { stress }\end{array}$ & $\begin{array}{c}\text { Severe } \\
\text { stress }\end{array}$ & $\begin{array}{c}\text { Adequate } \\
\text { water supply }\end{array}$ & $\begin{array}{c}\text { Moderate } \\
\text { stress }\end{array}$ & & $\begin{array}{c}\text { Adequate } \\
\text { water supply }\end{array}$ & $\begin{array}{c}\text { Moderate } \\
\text { stress }\end{array}$ & $\begin{array}{c}\text { Severe } \\
\text { stress }\end{array}$ \\
\hline \multicolumn{10}{|c|}{ Genetic parameters } \\
\hline$\overline{\mathrm{D}}$ & 0.06 & $11.36^{\text {*** }}$ & $3.71^{*}$ & $2.02^{* * \pi}$ & $2.81^{*}$ & $1.71^{* \cdots \times}$ & $0.024^{\text {**F }}$ & $0.032^{\text {**a }}$ & $0.012^{\text {*** }}$ \\
\hline $\mathrm{H}_{1}$ & $6.09^{*}$ & 4.80 & 7.62 & $5.16^{*}$ & 4.70 & 0.84 & 0.004 & $0.013^{* *}$ & 0.0002 \\
\hline $\mathrm{H}_{2}$ & $4.99^{*}$ & 4.36 & 6.66 & $4.73^{*}$ & 2.78 & 0.11 & 0.003 & $0.008^{* * *}$ & 0.0002 \\
\hline $\mathrm{F}$ & -0.41 & 1.03 & -6.79 & -0.04 & 2.81 & -2.21 & $0.010^{* *}$ & $0.016^{* * *}$ & -0.006 \\
\hline$h^{2}$ & $3.17^{*}$ & $9.30^{*}$ & $7.76^{* *}$ & $4.92^{* *}$ & 2.43 & -0.28 & 0.001 & $0.010^{* * *}$ & $0.001^{* *}$ \\
\hline$\underline{E}$ & $1.69^{* *}$ & $5.01^{* *}$ & $1.69^{* *}$ & 0.56 & $1.57^{* * *}$ & $1.55^{* * *}$ & $0.001^{* * *}$ & 0.001 & $0.0004^{* * *}$ \\
\hline \multicolumn{10}{|c|}{ Derived parameters } \\
\hline$\left[\mathrm{H}_{1} / \mathrm{D}\right]^{0.5}$ & 10.18 & 0.65 & 1.43 & 1.60 & 1.29 & 0.70 & 0.41 & 0.64 & 0.14 \\
\hline$\left[\mathrm{H}_{2} / 4 \mathrm{H}_{1}\right]$ & 0.20 & 0.23 & 0.22 & 0.23 & 0.15 & 0.03 & 0.16 & 0.15 & 0.22 \\
\hline$\left[\mathrm{h}^{2} / \mathrm{H}_{2}\right]$ & 0.64 & 2.13 & 1.16 & 1.04 & 0.87 & -2.66 & 0.45 & 1.26 & 3.00 \\
\hline [ KD / KR ] & 0.49 & 1.15 & 0.22 & 0.99 & 2.26 & 0.04 & 3.32 & 2.33 & 0.67 \\
\hline $\mathrm{T}_{(\mathrm{n})}$ & 21.13 & 46.88 & 63.08 & 41.70 & 29.70 & 59.67 & 83.68 & 79.96 & 92.99 \\
\hline
\end{tabular}

*** Significant at $\mathrm{P}=0.05$ and $\mathrm{P}=0.01$, respectively

\section{Yield and its attributes}

Data presented in Tables (8 and 9) indicated that the genetic components of variance and their derived parameters were fluctuated from condition to another, since the dominance genetic variance was significant and accounted the main type controlling the inheritance of head diameter, 100-achene weight and achene yield/plant under all environments, resulting in an average degree of dominance $\left(\mathrm{H}_{1} / \mathrm{D}\right)^{0.5}$ was more than unity.
Both additive (D) and dominance $\left(\mathrm{H}_{1}\right.$ and $\left.\mathrm{H}_{2}\right)$ genetic components were significant and involved in the inheritance of plant height, achene yield/plant, achene oil content under the three levels of water regimes; and head diameter under moderate and sever stress as well as 100-achene weight under severe stress, with the predominant of additive gene action in controlling plant height and achene oil content. Whereas, dominance gene action was the prevailed type governing head diameter and achene yield/plant under the three levels of water regimes. 


\section{J. Plant Production, Mansoura Univ., Vol. 7(12), December, 2016}

The covariance of additive and dominance gene effects in the parents ( $\mathrm{F}$ value) was positive and significant for plant height and achene yield/plant under the three levels of water regimes, indicating that increasing alleles were more frequent than the decreasing ones in the parental populations. Whereas, $\mathrm{F}$ value was negative and significant for head diameter under adequate water supply, as well as, 100- achene weight under both adequate water supply and moderate water stress, but in the remaining cases, the $F$ value didn't reach the level of significance.

The overall dominance effects of heterozygous loci $\left(h^{2}\right)$ were positive and significant for plant height, head diameter, achene yield/plant under the three levels of water regimes as well as 100-achene weight and achene oil content under adequate water supply and moderate water stress. The environmental variance was significant for yield contributing characters in most cases and achene oil content under moderate stress only. Whereas, it was insignificant under adequate water supply and severe stress conditions for that character, revealing that achene oil content was more heritable character and less influenced by the environmental fluctuations

The proportion of genes with positive and negative effects in the parents $\left(\mathrm{H}_{2} / 4 \mathrm{H}_{1}\right)$ was near to its maximum value $(0.25)$ for plant height under severe stress as well as achene oil content under both adequate water supply and moderate stress. The $\left(\mathrm{H}_{2} / 4 \mathrm{H}_{1}\right)$ was less than its maximum value $(0.25)$ for the remaining yield contributing characters under different levels of water regimes.

Table 8. Genetic components for morphological characters of sunflower genotypes of half- diallel analysis under three environments.

\begin{tabular}{|c|c|c|c|c|c|c|c|c|c|}
\hline \multirow{2}{*}{$\begin{array}{l}\text { Character } \\
\text { Water supply }\end{array}$} & \multicolumn{3}{|c|}{ Plant height $(\mathrm{cm})$} & \multicolumn{3}{|c|}{ Head diameter $(\mathrm{cm})$} & \multicolumn{3}{|c|}{ 100-achene weight (g) } \\
\hline & $\begin{array}{c}\text { Adequate } \\
\text { water supply }\end{array}$ & $\begin{array}{c}\text { Moderate } \\
\text { stress }\end{array}$ & $\begin{array}{l}\text { Severe } \\
\text { stress }\end{array}$ & $\begin{array}{c}\text { Adequate } \\
\text { water supply }\end{array}$ & $\begin{array}{c}\text { Moderate } \\
\text { stress }\end{array}$ & $\begin{array}{c}\text { Severe } \\
\text { stress }\end{array}$ & $\begin{array}{c}\text { Adequate } \\
\text { water supply }\end{array}$ & $\begin{array}{c}\text { Moderate } \\
\text { stress }\end{array}$ & $\begin{array}{c}\text { Severe } \\
\text { stress }\end{array}$ \\
\hline \multicolumn{10}{|c|}{ Genetic parameters } \\
\hline$\overline{\mathrm{D}}$ & $586.40^{3 * \pi}$ & $536.93^{* 3 x}$ & $267.61^{* / 2}$ & 1.29 & $1.98^{* * \pi}$ & $0.96^{3 \times 1 / 2}$ & $0.50^{\text {**a }}$ & $0.23^{*}$ & $0.63^{\text {** }}$ \\
\hline $\mathrm{H}_{1}$ & $341.61^{* *}$ & $359.86^{* *}$ & $159.04^{* *}$ & $6.88^{* *}$ & $3.34^{* *}$ & $1.64^{* * *}$ & $1.26^{* *}$ & $0.97^{* *}$ & $0.98^{* * *}$ \\
\hline $\mathrm{H}_{2}$ & $292.37^{* *}$ & $276.62^{* *}$ & $150.72^{* *}$ & $6.22^{* *}$ & $3.19^{* *}$ & $1.46^{* *}$ & $1.27^{* *}$ & $0.93^{* *}$ & 0.52 \\
\hline $\mathrm{F}$ & $271.53^{* * *}$ & $400.81^{* *}$ & $97.19^{* *}$ & -2.43 & 0.31 & -0.31 & -0.29 & -0.34 & $1.01^{* * *}$ \\
\hline$h^{2}$ & $655.78^{* *}$ & $444.93^{* *}$ & $295.68^{* *}$ & $15.10^{* *}$ & $4.49^{* *}$ & $1.02^{* * *}$ & $1.42^{* *}$ & $0.90^{* *}$ & 0.08 \\
\hline$\underline{E}$ & $20.14^{*}$ & 19.55 & $16.41^{*}$ & 0.46 & $0.40^{* *}$ & $0.26^{* * *}$ & $0.10^{* * *}$ & $0.18^{* * *}$ & $0.27^{* * *}$ \\
\hline \multicolumn{10}{|c|}{ Derived parameters } \\
\hline$\left[\mathrm{H}_{1} / \mathrm{D}\right]^{0.5}$ & 0.76 & 0.82 & 0.77 & 2.31 & 1.30 & 1.30 & 1.59 & 2.04 & 1.25 \\
\hline$\left[\mathrm{H}_{2} / 4 \mathrm{H}_{1}\right]$ & 0.21 & 0.19 & 0.24 & 0.23 & 0.24 & 0.22 & 0.25 & 0.24 & 0.13 \\
\hline$\left[\mathrm{h}^{2} / \mathrm{H}_{2}\right]$ & 2.24 & 1.61 & 1.96 & 2.43 & 1.41 & 0.69 & 1.12 & 0.97 & 0.15 \\
\hline [ KD / KR ] & 1.87 & 2.68 & 1.62 & 0.42 & 1.13 & 0.78 & 0.69 & 0.47 & 4.57 \\
\hline $\mathrm{T}_{(\mathrm{n})}$ & 66.13 & 55.29 & 62.30 & 52.12 & 43.29 & 53.72 & 48.66 & 42.83 & 9.89 \\
\hline
\end{tabular}

*** Significant at $\mathbf{P}=0.05$ and $\mathbf{P}=0.01$, respectively

Table 9. Genetic components for achene yield/head and achene oil content (\%) characters of sunflower genotypes of half-diallel analysis under three environments.

\begin{tabular}{|c|c|c|c|c|c|c|}
\hline \multirow[b]{2}{*}{$\begin{array}{l}\text { Character } \\
\text { Water supply }\end{array}$} & \multicolumn{3}{|c|}{ Achene yield /plant (g) } & \multicolumn{3}{|c|}{ Achene oil content (\%) } \\
\hline & $\begin{array}{c}\begin{array}{c}\text { Adequate water } \\
\text { supply }\end{array} \\
\end{array}$ & $\begin{array}{c}\text { Moderate } \\
\text { stress }\end{array}$ & $\begin{array}{c}\text { Severe } \\
\text { stress }\end{array}$ & $\begin{array}{c}\text { Adequate water } \\
\text { supply }\end{array}$ & $\begin{array}{c}\begin{array}{c}\text { Moderate } \\
\text { stress }\end{array} \\
\end{array}$ & $\begin{array}{r}\text { Severe } \\
\text { stress } \\
\end{array}$ \\
\hline \multicolumn{7}{|c|}{ Genetic parameters } \\
\hline$\overline{\mathrm{D}}$ & $109.53^{\text {*** }}$ & $99.46^{* \% \pi}$ & $28.78^{* * \pi}$ & $16.60^{\text {*कF }}$ & $15.90^{\text {**F }}$ & $14.98^{* * *}$ \\
\hline $\mathrm{H}_{1}$ & $155.19^{* *}$ & $167.82^{*}$ & 44.30 & $8.33^{* *}$ & $4.75^{* *}$ & $5.76^{*}$ \\
\hline $\mathrm{H}_{2}$ & $133.59^{* *}$ & 119.31 & 31.57 & $7.74^{* *}$ & $4.64^{* *}$ & $4.82^{* *}$ \\
\hline $\mathrm{F}$ & $99.02^{*}$ & 130.96 & 40.10 & 0.02 & -0.11 & 1.63 \\
\hline$h^{2}$ & $97.47^{* *}$ & 29.51 & $29.18^{*}$ & $10.70^{* * *}$ & $4.29^{* * *}$ & 1.97 \\
\hline $\mathrm{E}$ & $25.32^{* * *}$ & 15.16 & $14.32^{* *}$ & 0.36 & $0.38^{*}$ & 0.35 \\
\hline \multicolumn{7}{|c|}{ Derived parameters } \\
\hline$\left[\mathrm{H}_{1} / \mathrm{D}\right]^{0.5}$ & 1.19 & 1.30 & 1.24 & 0.71 & 0.55 & 0.62 \\
\hline$\left[\mathrm{H}_{2} / 4 \mathrm{H}_{1}\right]$ & 0.22 & 0.18 & 0.18 & 0.23 & 0.24 & 0.21 \\
\hline$\left[\mathrm{h}^{2} / \mathrm{H}_{2}\right]$ & 0.73 & 0.25 & 0.92 & 1.38 & 0.92 & 0.41 \\
\hline$[\mathrm{KD} / \mathrm{KR}]$ & 2.22 & 3.06 & 3.56 & 1.00 & 0.99 & 1.19 \\
\hline $\mathrm{T}_{(\mathrm{n})}$ & 21.48 & 15.90 & 3.07 & 78.90 & 84.01 & 82.14 \\
\hline
\end{tabular}

*,** Significant at $\mathbf{P}=0.05$ and $\mathbf{P}=0.01$, respectively

The proportion of dominance to recessive genes in the parents $(\mathrm{KD} / \mathrm{KR})$ was around the unity for achene oil content, while it was more than unity for achene yield / plant and plant height under different levels of water supply. On the other wise, excess of recessive alleles have been detected for head diameter under the three levels of water regimes as well as 100-achene weight under both adequate water supply and moderate stress.

Narrow sense heritability was high $(>50 \%)$ for plant height, and achene oil content under various levels of water regimes and head diameter under adequate water supply and severe water stress. Moderate narrow sense heritability have been registered for head diameter under moderate water regime; 100-achene weight under adequate water supply and moderate water regime. Whereas, low narrow sense heritability was recorded for 100 -achene weight under severe water stress and achene yield/plant under the three levels of water regimes.

Therefore, selection for the aforementioned characters must extended for late segregating generations. Hervé et al. (2001) indicated that the traits related to photosynthetic activity and water status are genotype 
dependent in sunflower. Narrow sense heritability was low for relative water content $(0.22)$, moderate for transpiration (0.40), and high for chlorophyll concentration (0.57). However, Ortis et al. (2005) indicated the predominant role of additive component for plant height, 1000-seed weight and seed oil content. Thus, hybrid breeding method could be used aiming to improve these characters. On the other hand, Ghaffari et al. (2011) indicated that 1000-seed weight, and oil yield were under control of both additive and dominance effects, plant height and oil content were controlled by additive effects, however over dominance effects were detected for seed yield.

Phenotypic, genotypic and environmental correlation coefficients

Phenotypic, genotypic and environmental correlation coefficients were used to determine the most effective characters which played an important role in the final yield across the three environments (Table 10). Positive and significant correlations were registered between achene yield/plant and each of leaf water content, transpiration rate, plant height, head diameter and 100-achene weight. These results are hold true at both phenotypic and genotypic levels, except for leaf water content which showed significant correlation at genotypic level only. Whereas, significantly negative genotypic and phenotypic correlations were recorded between achene oil content and achene yield/plant. Therefore, increasing values of achene oil content resulted in decreasing achene yield/plant.

Positive and highly significant environmental correlation was observed between 100-achene weight and achene yield/plant. This result could be discussed on the basis that both characters having the same environmental requirements. Moreover, positive and highly significant interrelationship was recorded between leaf water content with each of leaf chlorophyll content, transpiration rate and 100 -achene weight, and between transpiration rate with each of plant height, head diameter and 100-achene weight, as well as, between plant height and both head diameter and 100-achene weight at both genotypic and phenotypic levels in most cases. Hereby, increasing value of one character led to increase the another associated character. On the contrary, negative and highly significant association was registered between leaf water content with achene oil content and between leaf chlorophyll content with each of transpiration rate, plant height and head diameter as well as, between transpiration rate, plant height, head diameter on one hand with achene oil content, on the other hand. These results are in agreement with a few exceptions with those recorded by Darvishzadeh et al. (2011), Rauf et al. (2012), Ardiarini et al. (2013) and Iqbal et al. (2013).

Table 10. Genotypic (G), phenotypic (P) and environmental (e) correlation coefficients of various metric traits of sunflower genotypes across three environments.

\begin{tabular}{|c|c|c|c|c|c|c|c|c|}
\hline Characters & & $\begin{array}{l}\text { Leaf chlorophyll } \\
\text { content }(\%)\end{array}$ & $\begin{array}{c}\text { Transpiration } \\
\text { rate }\end{array}$ & $\begin{array}{l}\text { Plant height } \\
(\mathrm{cm})\end{array}$ & $\begin{array}{c}\text { Head } \\
\text { diameter }\end{array}$ & $\begin{array}{l}\text { 100- achene } \\
\text { weight }(g)\end{array}$ & $\begin{array}{c}\text { Achene oil } \\
\text { content }(\%)\end{array}$ & $\begin{array}{c}\text { Achene yield/ } \\
\text { plant }\end{array}$ \\
\hline \multirow{3}{*}{$\begin{array}{l}\text { Leaf water content } \\
(\%)\end{array}$} & $\mathrm{rg}$ & $0.452 * *$ & $0.236 *$ & 0.055 & -0.070 & $0.615 * *$ & $-0.405 * *$ & $0.381 * *$ \\
\hline & rp & 0.166 & 0.171 & 0.064 & -0.100 & $0.219 *$ & $-0.259 *$ & 0.179 \\
\hline & re & -0.063 & 0.082 & 0.112 & -0.168 & -0.139 & 0.102 & -0.002 \\
\hline \multirow{3}{*}{$\begin{array}{l}\text { Leaf chlorophyll } \\
\text { content }\end{array}$} & $\mathrm{rg}$ & 1 & $-0.255^{*}$ & $-0.589 * *$ & $-0.256^{*}$ & -0.142 & -0.144 & -0.019 \\
\hline & $\mathrm{rp}$ & 1 & -0.181 & $-0.414 * *$ & -0.190 & -0.085 & -0.085 & 0.041 \\
\hline & re & 1 & -0.090 & -0.191 & -0.117 & -0.035 & 0.071 & 0.092 \\
\hline \multirow{3}{*}{ Transpiration rate } & $\mathrm{rg}$ & & 1 & $0.668 * *$ & $0.601 * *$ & $0.576^{* *}$ & $-0.617 * *$ & $0.674 * *$ \\
\hline & $\mathrm{rp}$ & & 1 & $0.584 * *$ & $0.506 * *$ & $0.366 * *$ & $-0.569 * *$ & $0.476 * *$ \\
\hline & re & & 1 & 0.061 & 0.092 & -0.029 & -0.097 & 0.148 \\
\hline \multirow{3}{*}{ Plant height } & rg & & & 1 & $0.679 * *$ & $0.340 * *$ & $-0.307 * *$ & $0.653 * *$ \\
\hline & $\mathrm{rp}$ & & & 1 & $0.566 * *$ & $0.239 *$ & $-0.279 * *$ & $0.414 * *$ \\
\hline & re & & & 1 & 0.055 & 0.065 & 0.068 & -0.044 \\
\hline \multirow{3}{*}{ Head diameter } & $\mathrm{rg}$ & & & & 1 & 0.097 & $-0.291 * *$ & $0.577 * *$ \\
\hline & $\mathrm{rp}$ & & & & 1 & 0.101 & $-0.249 *$ & $0.378 * *$ \\
\hline & re & & & & 1 & 0.121 & 0.047 & 0.067 \\
\hline \multirow{3}{*}{ 100-achene weight } & $\mathrm{rg}$ & & & & & 1 & -0.117 & $0.311 * *$ \\
\hline & $\mathrm{rp}$ & & & & & 1 & -0.071 & $0.298 * *$ \\
\hline & re & & & & & 1 & 0.085 & $0.285 * *$ \\
\hline \multirow{3}{*}{$\begin{array}{l}\text { Achene oil content } \\
(\%)\end{array}$} & $\mathrm{rg}$ & & & & & & 1 & $-0.563 * *$ \\
\hline & $\mathrm{rp}$ & & & & & & 1 & $-0.390 * *$ \\
\hline & re & & & & & & 1 & -0.007 \\
\hline
\end{tabular}

*,** Significant at $\mathbf{P}=0.05$ and $\mathbf{P}=0.01$, respectively

\section{Path coefficient analysis}

Maximum direct effect on achene yield/plant was accounted for transpiration rate and plant height with values of $12.941 \%$ and $12.219 \%$. Whereas, moderate direct effects were recorded by both 100 -achene weight and leaf water content with values of 7.128 and $7.779 \%$. Moreover, the other remaining three characters i.e., leaf chlorophyll content, achene oil content and head diameter were less contribution as exhibited 1.917, 0.438 and $0.081 \%$, respectively. The highest indirect effects on achene yield/plant variation were observed for transpiration rate via plant height followed by transpiration rate via 100achene weight, leaf water content via 100 -achene weight, plant height via 100 -achen and leaf chlorophyll content via plant height with values of 8.442, 5.530, 4.579, 3.181 and 2.858, respectively (Table 11 ).

According to the total contribution of the studied characters on achene yield/plant variation, it could be arranged as follows, transpiration rate $(22.778 \%)$ plant height (20.413\%), 100-achene weight (13.939\%), leaf water content $(12.796 \%)$, leaf chlorophyll content (4.997\%), achene oil content $(2.571 \%)$ and then head diameter $(0.871 \%)$. Generally, it could be concluded that the studied characters accounted for $78.365 \%$ of the achene yield/plant variation, however the residual effect was $21.635 \%$. Hence, the results obtained from correlation and path analyses 
revealed the importance of transpiration rate, plant height, 100 -achene weight and leaf water content as selection criteria for improvement of sunflower yield. Hence, the results obtained from correlation and path analyses revealed the importance of transpiration rate, plant height, 100-achene weight and leaf water content as selection criteria for improvement of sunflower yield. Similar results were registered by Rauf et al. (2012), Ardiarini et al. (2013) and Iqbal et al. (2013). Also, Darvishzadeh et al. (2011) indicated that genotypic correlations manifest that seed yield per plant was positively and significantly associated with head diameter, plant height, number of leaf and achene traits under well-watered condition. While, under the waterstressed state, head weight, head diameter, number of achene and chlorophyll content showed positive and significant correlation with seed yield per plant. Head diameter and number of achene under both conditions and chlorophyll content under water-stressed condition have positive direct effect on seed yield/plant.

Table 11. Direct and indirect effect of various metric traits of sunflower genotypes on achene yield/ plant across three environments

\begin{tabular}{|c|c|c|c|}
\hline S.O.V. & CD & RI \% & $\begin{array}{c}\text { Total contribution } \\
\text { on achene } \\
\text { yield/plant }\end{array}$ \\
\hline Leaf water content $\% \quad\left(\mathrm{X}_{1}\right)$ & 0.07779 & 7.779 & 12.796 \\
\hline Leaf chlorophyll content $\quad\left(X_{2}\right)$ & 0.01917 & 1.917 & 4.997 \\
\hline Transpiration rate & 0.12941 & 12.941 & 22.778 \\
\hline Plant height & 0.12219 & 12.219 & 20.413 \\
\hline Head diameter & 0.00081 & 0.081 & 0.871 \\
\hline 100 -achene weight & 0.07128 & 7.128 & 13.939 \\
\hline Achene oil content $\% \quad\left(\mathrm{X}_{7}\right)$ & 0.00438 & 0.438 & 2.571 \\
\hline $\mathrm{X}_{1} \times \mathrm{X}_{2}$ & 0.01746 & 1.746 & \\
\hline $\mathrm{X}_{1} \times \mathrm{X}_{3}$ & 0.02367 & 2.367 & \\
\hline $\mathrm{X}_{1} \times \mathrm{X}_{4}$ & 0.00537 & 0.537 & \\
\hline $\mathrm{X}_{1} \times \mathrm{X}_{5}$ & 0.00056 & 0.056 & \\
\hline $\mathrm{X}_{1} \times \mathrm{X}_{6}$ & 0.04579 & 4.579 & \\
\hline $\mathrm{X}_{1} \times \mathrm{X}_{7}$ & 0.00748 & 0.748 & \\
\hline$X_{2} \times X_{3}$ & 0.01270 & 1.270 & \\
\hline $\mathrm{X}_{2} \times \mathrm{X}_{4}$ & 0.02858 & 2.858 & \\
\hline $\mathrm{X}_{2} \times \mathrm{X}_{5}$ & 0.00101 & 0.101 & \\
\hline $\mathrm{X}_{2} \times \mathrm{X}_{6}$ & 0.00525 & 0.525 & \\
\hline $\mathrm{X}_{2} \times \mathrm{X}_{7}$ & 0.00132 & 0.132 & \\
\hline $\mathrm{X}_{3} \times \mathrm{X}_{4}$ & 0.08422 & 8.422 & \\
\hline$X_{3} \times X_{5}$ & 0.00615 & 0.615 & \\
\hline $\mathrm{X}_{3} \times \mathrm{X}_{6}$ & 0.05530 & 5.530 & \\
\hline $\mathrm{X}_{3} \times \mathrm{X}_{7}$ & 0.01470 & 1.470 & \\
\hline $\mathrm{X}_{4} \times \mathrm{X}_{5}$ & 0.00678 & 0.678 & \\
\hline $\mathrm{X}_{4} \times \mathrm{X}_{6}$ & 0.03181 & 3.181 & \\
\hline $\mathrm{X}_{4} \times \mathrm{X}_{7}$ & 0.00713 & 0.713 & \\
\hline $\mathrm{X}_{5} \times \mathrm{X}_{6}$ & 0.00074 & 0.074 & \\
\hline $\mathrm{X}_{5} \times \mathrm{X}_{7}$ & 0.00055 & 0.055 & \\
\hline $\mathrm{X}_{6} \times \mathrm{X}_{7}$ & 0.00207 & 0.207 & \\
\hline $\mathrm{R}^{2}$ & 0.78365 & 78.365 & 78.365 \\
\hline Residual & 0.21635 & 21.635 & 21.635 \\
\hline Total & 1.00000 & 100 & 100.000 \\
\hline
\end{tabular}

\section{REFERENCES}

AOAC (1984). Official method of Analysis, Association of Official Analytical Chemist (AOAC) Washington D.C., USA, pp: 1250-1255.

Ardiarini, R. N. and K. Kuswanto (2013). The path analysis on yield due to the sunflower's (Helianthus annuus L.) oil under drought stress. J. Basic. Appl. Sci. Res. , 3(4)1-7.
Bakheit, B.R.; A. M., Mahmoud; A.A. El-Shimy and M.A. Attia (2010). Combining ability for yield and yield components in sunflower. Egypt J. Plant Breed., 14(1):173-186.

Burli, A.V.; B.B. Pawar and M.G. Jadhav (2001). Combining ability studies of some mal sterile lines and restore in sunflower. J. Maharashtra Agric. Univ., 26(2):190-191.

Darvishzadeh, R. ; H. H. Maleki and A. Sarrafi (2011). Path analysis of the relationships between yield and some related traits in diallel population of sunflower (Helianthus annuus L.) under well-watered and water-stressed conditions. AJCS, 5(6):674-680.

Dewey, D. R. and K .H. Lu (1959). A correlation and path coefficient analysis of components of crested wheat grass production. Agron. J., 51: 515-518.

Encheva, J.; G. Georgiev and E. Penchev (2015). Heterosis effects for agronomically important traits in sunflower (Helianthus annuus L.). Bulg. J. Agric. Sci., 21: 336-341.

FAOSTAT, (2014). Food and Agricultural Organization Statistical Database. http://www.fa.org/ faostat/ en/ \#data/QC.

Fernández-Martínez, J. M.;B. Pérez-Vich and L. Velasco (2009). Sunflower. In Oil Crops; Vollmann, J., Rajcan, I., Eds.; Springer: New York, 2009; PP155232.

Fischer, R. and P. Maurer (1978). Drought resistance in spring wheat cultivars. I. Grain yield responses. Aust. J. Agric. Res., 29: 897-905.

Ghaffari, M.; I. Farokhi and M. Mirzapour (2011). Combining ability and gene action for agronomic traits and oil content in sunflower (Helianthus annuuns L.) using F1 hybrids. Crop Breeding J., 1 (1): $75-87$.

Gill, H.S.; S.R. Khurana; T.P. Yadava and R.K. Sheoran (1998). Expression of heterosis for different characters in sunflower over environments. Haryana Agric. University J. Res., 28: 95-100.

Goksoy, A. T. and Z.M. Turan (2004). Combining abilityties of certain characters and estimation of hybrid vigour in sunflower (Helianthus annuus L.). Acta Agronomica Hungarica, 52 (4): 361- 368.

Gosev, N.A. (1960). Some methods in studying plant water relations, Leningrade Acad of Sciena, USSR.

Guidi, L. and G.F. Soldatini (1997). Chlorophyll fluorescence and gas exchanges in flooded soybean and sunflower plants. Plant Physiology and Biochemistry, 35: 713-717.

Habib, H.; S.S. Mehdi; A. Rashid; M. Zafar and M.A. Anjum (2007). Heterosis and heterobeltiosis studies for flowering traits, plant height and seed yield in sunflower (Helianthus annuus L.). Int. J. Agri. Biol., 9(2): 355-358.

Hassan, M. B. and F. A. Sahfiqu (2010). Current situation of edible vegetable oils and some propositions to curb the oil gap in Egypt. Nature and Science, 8: 1-12.

Hayman, B. I. (1954a).The analysis of diallel tables. Biometrics, 10: 235-244.

Hayman, B. I. (1954b). The theory and analysis of diallel crosses. Genetics, 39: 789-809. 
Hervé, D.; F. Fabre; E. F. Berrios; N. Leroux; Gh. Al Chaarani; C. Planchon and A. Sarrafi (2001). QTL analysis of photosynthesis and water status traits in sunflower (Helianthus annuus L.) under greenhouse conditions. J. of Experimental Botany, 52 (362): 1857-1864.

Hirasawa, T; K. Wakabayashi; S. Touya and K. Ishihara (1995). Stomatal responses to water deficits and abscisic acid in leaves of sunflower plants (Helianthus annuus L.) grown under different conditions. Plant Cell Physiology, 36: 955-964.

Ibrahim, M.E.; E.A. El-Absawy; A.H. Selim and N.A. Gaafar (2003). Effect of nitrogen and phosphorous pigments, yield and yield attributes of some sunflower varieties (Helianthus annuus L.). Zagazig J. Agric. Res., 30 (4): 1223 - 1271.

Iqbal, M.; U. Ijaz; Smiullah M. Iqbal; K. Mahmood; M. Najeebullah; Abdullah;S. Niazand and H. A. Sadaqat (2013). Genetic divergence and path coefficient analysis for yield related attributes in sunflower (Helianthus annuus L.) under less water conditions at productive phase. Plant Knowledge Journal, 2(1): 20- 23.

Maury, P.; M. Berger; F. Mojayad and C. Planchon (2006). Leaf water characteristics and drought acclimation in sunflower genotypes. Plant and Soil, 223 (1-2): 155-162

Migahid, A.M. and F.A. Amer (1950). Three types of transpiration of snap bean. Agron. J., 44:562- 568.

Miller, J. F. (1998). Oilseed and heterosis. TEKTRAN. United States, Department of Agriculture. Agriculture Research Service. $62-80 \mathrm{p}$.

Miller, P. A.; J.C. Williams; H. F. Robinson and R. E. Comstock. (1958). Estimates of genotypic and environmental variance and covariance in Upland cotton and their implication in selection. Agron. J., $50: 126-131$.

Ortis, L.; G. Nestares; E. Frutos and N. Machado (2005). Combining ability Analysis for agronomic traits in sunflower (Helianthus annuus L.). Helia 28 (43): 125- 134
Pankovic D.; Z. Sakac; S. Kevresan and M. Plesnicar (1999). Acclimation to long term water deficit in the leaves of two sunflower hybrids: photosynthesis, electron transport and carbon metabolism. Journal of Experimental Botany, 50: 127-138.

Pourmohammad, A.; M. Toorchi; S. S Alavikia and M. $R$ Shakiba (2016). Estimation of genetic parameters for yield and yield components in sunflower under normal and stress water deficit. Bulg. J. Agric. Sci., 22: 426-430

Rauf, A. ; F. A. Khan and M. Aslam (2012). Genetic association and path coefficient analysis for biomass production in sunflower at different salinity levels . J. Anim. Plant Sci., 22(4): 1150-1155.

Rauf, S. and H. A. Sadaqat (2007). Screening sunflower (Helianthus annuus L.) germplasm for drought tolerance. Communications in Biometry and Crop Science 2(1): 8-16.

Ravi, R.; R. K. Sheoran; k. Rakesh and H. S. Gill (2004). Combining ability analysis in sunflower (Helianthus annuus L.). National J. Plant Improvement, 6 (2): 8993.

Reddy, A.R.; K.V. Chaitanya and M. Vivekanandan (2004). Drought-induced responses of photosynthesis and antioxidant metabolism in higher plants. J Plant Physiol. 161: 1189-1202.

Steel, R. G. D. and J. H. Torrie (1980). Principles and Procedures of Statistics: a Biometrical Approach. Second Edition. New York: Mc Graw-Hill Kogakusha.

Turner, N. C. (1981). Techniques and experimental approaches for the measurement of plant water status. Plant and Soil, 58:339-366.

Virgona, J.M.; K.T. Hubick; H.M. Rawson; G.D. Farquhar and R.W. Downes (1990). Genotypic variation in transpiration efficiency, carbon isotope discrimination and carbon allocation during early growth in sunflower. Australian Journal of Plant Physiology, 17: 207-214.

Wynne, J.C.; D.A. Emery and P.M. Rice (1970). Combining ability estimates in Arachis hypogaea L. II. Field performance of $F_{1}$ hybrids. Crop Sci., 10(6): 713-715.

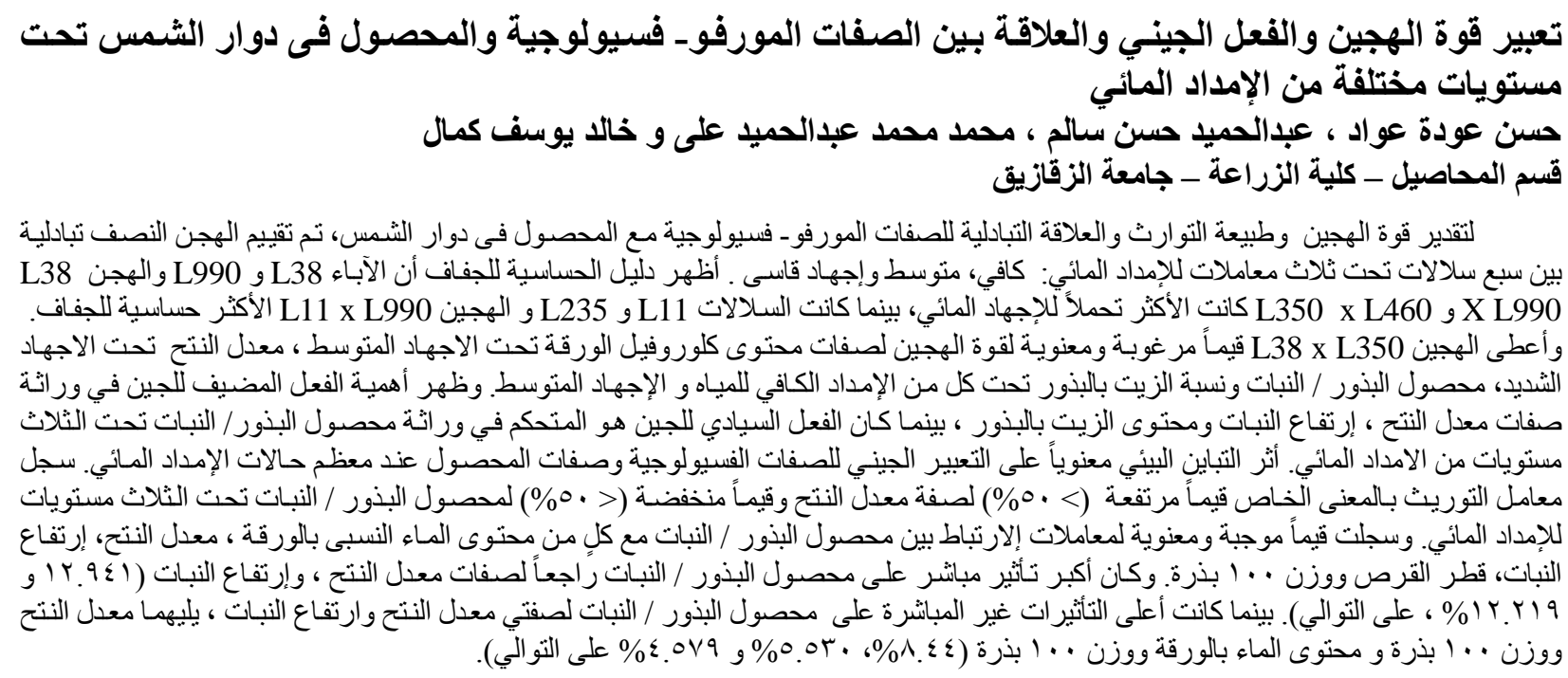

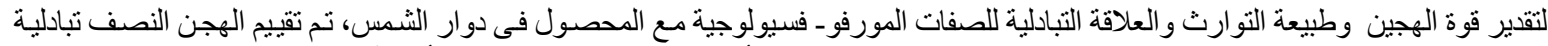

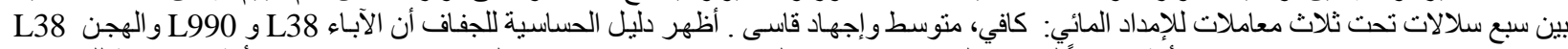

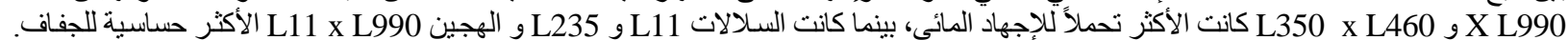

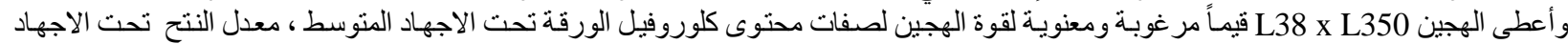

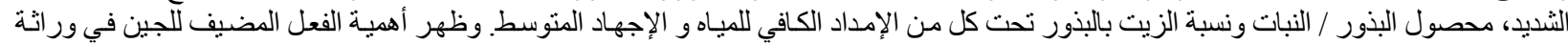

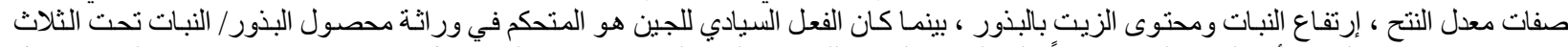

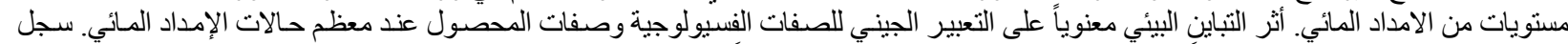

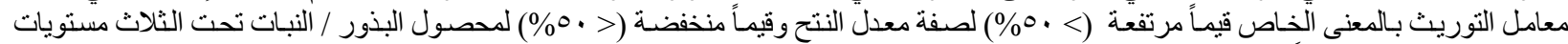

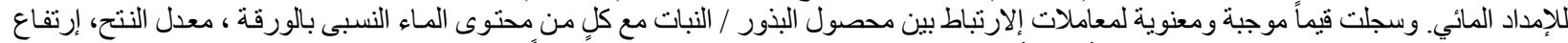

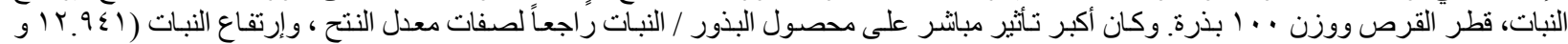

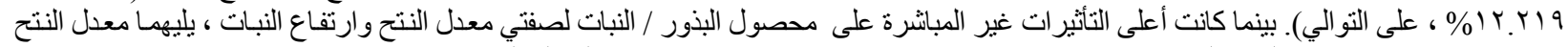

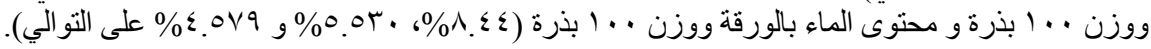

